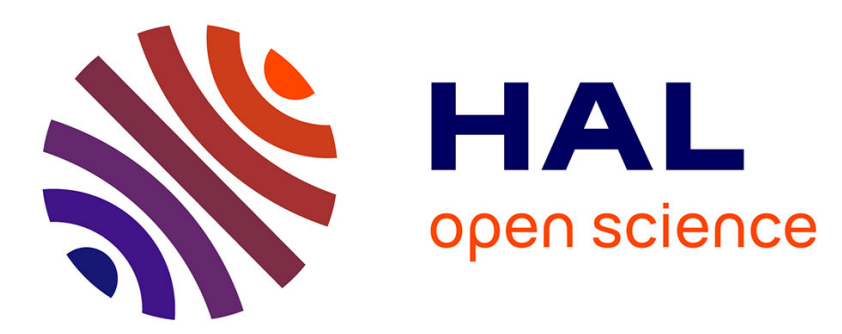

\title{
La conversation de marque: pratiques linguistiques sur les médias sociaux selon la théorie du face-work
}

\author{
Andria Andriuzzi, Géraldine Michel
}

\section{To cite this version:}

Andria Andriuzzi, Géraldine Michel. La conversation de marque: pratiques linguistiques sur les médias sociaux selon la théorie du face-work. Recherches et applications en Marketing, 2021, 10.1177/0767370120962610 . hal-03021314

\section{HAL Id: hal-03021314 https://hal.science/hal-03021314}

Submitted on 24 Nov 2020

HAL is a multi-disciplinary open access archive for the deposit and dissemination of scientific research documents, whether they are published or not. The documents may come from teaching and research institutions in France or abroad, or from public or private research centers.
L'archive ouverte pluridisciplinaire HAL, est destinée au dépôt et à la diffusion de documents scientifiques de niveau recherche, publiés ou non, émanant des établissements d'enseignement et de recherche français ou étrangers, des laboratoires publics ou privés. 


\title{
La conversation de marque : pratiques linguistiques sur les médias sociaux selon la théorie du face-work
}

\author{
Andria Andriuzzi, Coactis, Université Jean Monnet Saint-Etienne, France \\ Géraldine Michel, IAE Paris - Sorbonne, Université Paris 1 Panthéon-Sorbonne, France
}

Résumé : L'essor des médias sociaux a favorisé les échanges entre les internautes et les représentants des marques. Pour mieux comprendre comment cette « conversation de marque » se déroule, notre recherche mobilise la théorie du face-work et explore les perceptions ambivalentes des consommateurs vis-à-vis des pratiques linguistiques des marques. En particulier, nos résultats montrent comment des messages «menaçants » (selon la théorie du face-work) peuvent être acceptés par les consommateurs, tandis que des messages «valorisants » peuvent être rejetés. Ces situations paradoxales peuvent être expliquées par la nature de la relation à la marque, la dimension symbolique de la marque et le contexte commercial des échanges. En identifiant les spécificités des interactions marquesconsommateurs à la lumière du face-work, nous proposons un outil d'aide à la décision dans les domaines de la gestion de la marque et du community management.

Mots-clés : conversation, digital, face-work, marques, médias sociaux, normes

\section{Brand conversation: Linguistic practices on social media in the light of face-work theory}

\begin{abstract}
The rise of social media has resulted in brand-consumer interaction and more frequent conversations between consumers and brand representatives. To better understand how this "brand conversation" occurs, our research employs face-work theory and explores ambivalent consumer perceptions towards brand linguistic practices. Specifically, our results show how "threatening" messages (according to face-work theory) can be accepted by consumers, while "flattering" messages can be rejected. These paradoxical situations can be explained by brand relationships and the commercial and symbolic brand status. By identifying the specific features of brand-consumer interactions in the light of face-work, we propose a decision-making tool for brand management and community management practices.
\end{abstract}

Keywords: conversation, digital, face-work, brands, social media, norms

Pour citer cet article :

Andriuzzi, A., \& Michel, G. (2021). La conversation de marque : pratiques linguistiques sur les médias sociaux selon la théorie du face-work. Recherche et Applications En Marketing (French Edition) https://doi.org/10.1177/0767370120962610 


\section{La conversation de marque : pratiques linguistiques sur les médias sociaux selon la théorie du face-work}

\section{Introduction}

Dans l'objectif de créer des relations fortes avec les consommateurs, les entreprises développent la présence des marques sur des médias sociaux comme Facebook ou Twitter (Hamilton et al., 2016). Tout en constituant un nouveau canal auquel peuvent être assignés des objectifs, ces outils offrent la possibilité aux marques de communiquer de manière plus horizontale, en s'imbriquant dans des réseaux de relations interpersonnelles (Fournier et Avery, 2011). Une forme de conversation peut alors se développer, à l'initiative des marques ou des consommateurs. Nous définissons ainsi la conversation de marque comme une suite de messages échangés en ligne et en public entre plusieurs individus, dont l'un représente une marque et agit ouvertement en tant que tel. Elle peut se dérouler dans des espaces gérés par les marques, tels que leur compte Instagram, ou dans des espaces en ligne maîtrisés par les internautes, comme par exemple des forums de consommateurs.

Face à la multiplicité de ces situations il est important, pour les chercheurs comme pour les praticiens, d'identifier les leviers d'une conversation de marque réussie. Plusieurs questions restent en effet en suspens : quels modèles conversationnels sont utilisés par les marques sur les médias sociaux ? Comment ces modèles conversationnels sont-ils perçus par les internautes ? Répondre à ces questions permettrait de mieux comprendre comment la conversation en ligne des marques peut engager les consommateurs dans des interactions et des relations avec les marques. De tels travaux feraient ainsi écho aux appels à de nouvelles recherches sur le contenu des interactions marques-consommateurs en ligne (ex. : Villarroel Ordenes et al., 2019 ; Smith et Rose, 2020).

Pour analyser les conversations en ligne entre marques et consommateurs, des recherches ont posé l'idée que la conversation de marque pouvait être considérée comme un élément d'expression de la marque guidé par ses valeurs et cohérent avec sa personnalité (Michel, 2017). Dans ce contexte, il a été montré que les marques qui utilisent des pronoms personnels génèrent plus d'implication des consommateurs, par rapport aux marques qui n'en utilisent pas (Cruz et al., 2017). D'autres travaux révèlent que l'utilisation des émoticônes par les marques leur confère un caractère chaleureux ( $\mathrm{Li}$ et al., 2018), tandis que les consommateurs qui connaissent bien une marque lui témoignent plus de confiance lorsqu'elle utilise un langage informel (Gretry et al., 2017). Cependant, on sait peu de choses sur les règles linguistiques en vigueur dans la conversation en ligne des marques et sur la façon dont les internautes perçoivent le respect ou la transgression de ces règles par les marques. 
Dans la mesure où la conversation de marque implique généralement l'intervention d'un représentant humain, souvent un community manager, et donc la mise en scène de relations interpersonnelles (Griffiths et Mclean, 2015), cette recherche propose de mobiliser la théorie du face-work, qui permet d'analyser précisément les interactions interindividuelles, du point de vue des participants et des spectateurs. Le principe fondamental de la théorie du face-work, ou «effort de figuration » en français, est que, lors d'une interaction, les individus s'attachent à faire bonne figure tout en faisant en sorte que les autres ne perdent pas la face (Goffman, 1955). Les règles de conduite consistent alors à atténuer les actes pouvant porter atteinte à la face (face threatening acts; Brown et Levinson, 1987) et à maximiser les actes qui peuvent valoriser la face (face flattering acts ; Kerbrat-Orecchioni, 1998).

La marque n'étant pas un interlocuteur comme les autres, l'objectif de cette recherche est, dans une perspective exploratoire, de mieux comprendre dans quelle mesure les marques, en tant qu'entités symboliques, utilisent les règles du face-work dans leurs conversations en ligne et quels en sont les effets potentiels. Pour répondre à ces questions, nous avons mené une étude qualitative sous la forme d'une observation en ligne non participante, en analysant 151 conversations de six marques dans trois secteurs d'activité (automobile, alimentation et beauté), et conduit une série d'entretiens avec 12 consommateurs. Ces deux approches permettent, d'une part, d'analyser les pratiques conversationnelles des marques et les réactions des consommateurs qui participent aux conversations et, d'autre part, de connaître les perceptions des consommateurs vis-à-vis de ces conversations, qu'ils y participent ou qu'ils n'en soient que les spectateurs.

Dans un premier temps, nos résultats montrent que les marques utilisent différentes pratiques linguistiques en accord avec les règles du face-work. D'une part, elles peuvent chercher à minimiser une éventuelle perte de liberté chez leur interlocuteur en atténuant leurs face threatening acts (FTA). D'autre part, elles tentent de répondre au besoin de reconnaissance des internautes en produisant des face flattering acts (FFA). Dans un second temps, notre analyse révèle que la relation à la marque, la nature symbolique des marques et le contexte commercial des échanges avec les internautes expliquent comment les face threatening acts des marques peuvent être acceptés et comment les face flattering acts sont parfois rejetés. Nos conclusions enrichissent ainsi la littérature sur les interactions marques-consommateurs et étendent la théorie du face-work au contexte des conversations en ligne entre marques et consommateurs. Enfin, sur la base d'une typologie des conversations de marque, nous formulons des recommandations pour la gestion de la marque et la pratique du community management. 


\section{Cadre théorique}

\section{La conversation, un nouveau mode d'interaction pour les marques}

Cette recherche s'inscrit dans le champ de la littérature sur les interactions marquesconsommateurs, qui se basent sur trois dimensions principales : une communication à double sens, un échange en temps réel et un contrôle actif (Liu et Shrum, 2002). Les interactions en ligne entre marques et consommateurs intègrent différents comportements d'engagement entre les deux parties tels que les «likes», les partages et les commentaires. Parmi cette multiplicité d'interactions, nous définissons ici la conversation de marque comme une suite de messages échangés en ligne et en public entre plusieurs individus, dont l'un représente une marque et agit ouvertement en tant que tel, sous quelque forme que ce soit (logo, personnage de marque, porte-parole, etc.).

Notre définition inclut dans le périmètre de la conversation de marque les interactions verbales avec la marque (commentaires, discussions avec les avatars des marques) ; par ailleurs, elle en exclut les interactions non verbales (ex. : likes, partages), les interactions non publiques telles que les échanges privés avec les services client en ligne (voir Van Dolen et al., 2007) et les interactions où le rôle de la marque n'est pas clairement identifié, comme lorsque les internautes n'indiquent pas explicitement leur lien avec la marque (ex. : un influenceur qui ne dévoilerait pas un partenariat). Cette définition précise ainsi le périmètre de la conversation de marque, jusque-là souvent utilisée de façon générique dans le domaine du marketing (BerthelotGuiet, 2011 ; De Montety et Patrin-Leclère, 2011 ; Drouet, 2011) et permet d'appréhender de façon plus fine les différentes formes de conversation.

A partir de cette définition, et sur la base d'une revue de la littérature sur la conversation de marque (voir Annexe 1), nous proposons une typologie des différentes formes de conversation de marque selon deux critères : l'initiative et le lieu de la conversation (Tableau 1).

\section{[Insérer Tableau 1]}

Nous avons identifié ces critères dans la littérature, où ils sont utilisés généralement indépendamment l'un de l'autre. En ce qui concerne l'initiative de la conversation, des recherches ont par exemple montré que les contenus générés par les utilisateurs sur les médias sociaux sont adressés plus souvent aux marques proactives, c'est-à-dire celles qui initient les conversations, plutôt qu'aux marques non-proactives (Smith et al., 2012). En ce qui concerne le lieu de la conversation, des recherches abordent par exemple le fait que les espaces gérés par les 
marques favorisent les interactions marques-consommateurs, comparés aux communautés en ligne gérées par les consommateurs (Breitsohl et al. 2015).

Le croisement de ces critères permet d'identifier quatre formes de conversation : (1) La conversation d'une marque hôte-proactive correspond au fait que la marque lance un sujet de conversation au sein d'un espace qu'elle maitrise (ex.: sa page Facebook), offrant aux internautes la possibilité de lui répondre. (2) La conversation d'une marque hôte-réactive est une conversation à l'initiative des internautes dans un espace administré par la marque et qui appelle une réaction de la marque (ex. : site de co-création). (3) La conversation d'une marque invitée-proactive consiste pour les marques à intervenir au sein d'un espace communautaire géré par les consommateurs (ex. : communauté de consommation en ligne) ou par un tiers (ex : Tripadvisor), ou encore à interpeller des consommateurs qui s'expriment depuis leurs comptes sur les médias sociaux sans avoir explicitement sollicité la marque (ex: blog personnel, Instagram). (4) La conversation d'une marque invitée-réactive correspond aux conversations où les marques répondent à des avis ou des questions de consommateurs qui s'adressent à la marque depuis leur compte personnel (ex : Twitter).

\section{Les bénéfices et les risques de la conversation de marque}

Au-delà des recherches qui s'intéressent à la quantité d'interactions, on peut s'interroger sur les véritables bénéfices de ces conversations et sur les risques qu'elles peuvent présenter. Les consommateurs peuvent trouver une forme de gratification dans l'interaction avec les marques (Simon et Tossan, 2018). Les marques peuvent en effet leur offrir des opportunités de s'exprimer mais aussi de satisfaire leur ego (Smith et al., 2012). Plus particulièrement, la réponse des marques à des avis négatifs de consommateurs génère une certaine satisfaction chez ces derniers (Van Noort et Willemsen, 2012), d'autant plus quand le commentaire est personnalisé (Wang et Chaudhry, 2018). D'autres recherches ont aussi montré que la nature interactive des publications des marques a un impact positif sur l'intention des consommateurs de partager des informations (Labrecque, 2014). Plus globalement, la littérature montre que plus la marque interagit avec les consommateurs sur les médias sociaux, plus la relation à la marque, la rentabilité du client et les ventes sont améliorées (Kumar et al., 2016).

Toutefois, le succès des dispositifs interactifs des marques n'est pas garanti. Ainsi, les effets positifs de la conversation de marque sont à nuancer selon l'implication des individus envers les produits ou les sujets de conversation (Homburg et al., 2015). Par ailleurs, les réponses des marques qui paraissent automatisées peuvent engendrer des réactions négatives (Labrecque, 2014 ; Van Noort et Willemsen, 2012). De même, une trop forte participation des 
marques aux conversations des internautes peut être perçue comme intrusive et dégrader l'attitude envers la marque (Homburg et al., 2015).

Pour mieux comprendre comment les consommateurs réagissent à la conversation de marque, il parait essentiel d'analyser les différentes formes conversationnelles mises en place par les marques. Des recherches ont déjà montré que les marques qui utilisent des techniques d'humanisation basées sur le langage, comme l'insertion des pronoms personnels à la première personne (Chen et al., 2015) ou à la deuxième personne (Cruz et al., 2017), génèrent plus de participation et d'implication de la part des consommateurs par rapport aux marques qui n'en utilisent pas. D'autres travaux révèlent que l'utilisation des émoticônes par les marques leur confère un caractère chaleureux apprécié par les consommateurs (Li et al., 2018), tandis qu'un langage informel est plus apprécié qu'un langage formel par les consommateurs qui connaissent bien la marque (Gretry et al., 2017). Plus globalement, les messages des marques au contenu émotionnel ou informatif sont plus partagés par les consommateurs que les messages directifs (Villarroel Ordenes et al., 2019).

\section{Conversation de marque et théorie du face-work}

Malgré l'importance des recherches précédemment citées, on sait encore peu de choses sur les mécanismes interpersonnels mis en œuvre dans la conversation de marque. Plus particulièrement, Smith et Rose (2020) insistent sur la nécessité d'étudier le rôle des normes dans les relations marques-consommateurs en ligne. Ainsi, afin de mieux comprendre les pratiques conversationnelles des marques, nous mobilisons la théorie du face-work, qui permet l'étude détaillée de telles normes en mettant en évidence un « ordre de l'interaction » (Goffman, 1973). De plus, la théorie du face-work permet de prendre en compte à la fois les participants et les spectateurs de conversations qui ont lieu dans un espace public, à la vue de tous (KerssenGriep et al., 2008).

Issue de la sociologie et de la linguistique, la théorie du face-work a été élaborée par Goffman (1967) avant d'être notamment développée par Brown et Levinson (1987) et par Kerbrat-Orecchioni (2005). Elle repose sur l'idée fondamentale que, dans le cadre d'une interaction, les individus s'attachent à faire bonne figure, tout en faisant en sorte que les autres ne perdent pas la face (Goffman, 1955). Pour Goffman (1967: p. 9), la face correspond à "la valeur sociale positive qu'une personne revendique effectivement à travers la ligne d'action que les autres supposent qu'elle a adoptée au cours d'un contact particulier". Pour respecter l'ordre de l'interaction, c'est-à-dire pour faire en sorte que les interactions se déroulent de façon satisfaisante pour chacune des parties, les individus cherchent à assouvir deux besoins 
fondamentaux : être libres et être reconnus (Brown et Levinson, 1987). Pour parvenir à ce fragile équilibre, ils mettent en œuvre une gamme complexe de pratiques linguistiques, répertoriées dans une théorie de la politesse, qui consacre le face-work en principe universel de toute communication humaine (Brown et Levinson, 1987).

Face au besoin de liberté et d'autonomie des individus, les incitations, comme des ordres ou des suggestions, peuvent empiéter sur le territoire de l'autre. De même, des impositions comme des cadeaux ou des propositions peuvent mettre l'interlocuteur dans une situation d'obligation. La théorie du face-work met ainsi en évidence des actes menaçant la face de l'interlocuteur (face threatening acts, FTA ; Brown et Levinson, 1987). Toutefois, quand la production d'actes menaçants s'avère inévitable, les individus ont la possibilité d'atténuer leur portée négative, par exemple en utilisant une forme interrogative, en offrant plusieurs options ou en dépersonnalisant leurs propos (Brown et Levinson, 1987). Par ailleurs, pour assouvir le besoin de reconnaissance de leurs interlocuteurs, les individus peuvent chercher à «augmenter leur face », par exemple en leur adressant des compliments ou en se montrant sympathiques. Ces actes valorisant la face (face flattering acts, FFA ; Kerbrat-Orecchioni 2005) peuvent aussi consister à tenter de coopérer avec son interlocuteur en cherchant à l'inclure ou en faisant preuve d'optimisme.

L'application de ces stratégies d'interaction semble complexe : la réponse à un besoin de valorisation (ex. : offrir un cadeau) peut à la fois satisfaire ce besoin et menacer un désir d'autonomie. La coexistence de deux tendances antinomiques, intégration et indépendance, rend inévitable la dualité sémantique de toute interaction sociale (Goffman, 1967). C'est pourquoi les individus font aussi appel à des critères d'évaluation contextuels pour identifier le niveau de risque de la «perte de face » et définir la stratégie d'interaction adaptée à la situation (Figure 1). Pour Brown et Levinson (1987), ils prennent en compte le degré d'imposition de l'acte, c'est-àdire la «gravité » de la menace pour la face dans une culture donnée, ainsi que la distance sociale et l'éventuelle relation de pouvoir existant entre les interlocuteurs.

\section{[Insérer Figure 1]}

Plus récemment, des chercheurs ont étudié les manifestations du face-work dans les interactions en ligne, que ce soit au sein de groupes de discussion ou sur les médias sociaux (Bedijs et al., 2014). Par exemple, quand des internautes souhaitent adhérer à un groupe de discussion et qu'ils voient leur demande rejetée et critiquée, leur désir de reconnaissance est inassouvi, ce qui génère des sentiments négatifs (Chen, 2015). De même, les publications des internautes sur les médias sociaux peuvent entraver le désir d'autonomie de leurs amis, par 
exemple en leur adressant des conseils insistants et non sollicités (ex. : "Allez voir le dernier Tarentino !!!”). Les individus utilisent alors une stratégie d'évitement qui vise à sauver la face de leur interlocuteur (Mauney et Jeon, 2014).

Les marques s'exprimant généralement au travers d'un porte-parole humain (ex. : un community manager) et sur des plates-formes conçues avant tout pour les individus (Fournier et Avery, 2011), nous pouvons penser que les règles du face-work s'appliquent aussi dans ce contexte. Cependant, la marque n'étant pas un interlocuteur comme les autres, il est possible qu'apparaissent des spécificités dans l'application de ces règles. Cette recherche a donc pour objectif de mieux comprendre les pratiques conversationnelles des marques et la perception qu'en ont les consommateurs selon les règles linguistiques utilisées par les marques.

\section{Méthodologie}

Nous proposons d'étudier la conversation de marque à la lumière de la théorie du face-work, en considérant plusieurs types d'acteurs (marques, consommateurs participants et spectateurs), différents contextes (plusieurs médias sociaux) et plusieurs types de marques (produits et services; Tableau 2). Pour identifier les différentes pratiques linguistiques utilisées par les marques et pour étudier le comportement des internautes qui participent aux conversations, nous avons réalisé une observation non participante des conversations en ligne entre marques et internautes, basée sur la collecte de 151 conversations impliquant six marques dans trois secteurs d'activité. De plus, pour mieux comprendre les perceptions des consommateurs, qu'ils participent aux conversations ou qu'ils n'en soient que spectateurs, nous avons interrogé 12 consommateurs dans le cadre d'entretiens semi-directifs individuels.

\section{[Insérer Tableau 2]}

\section{Etude qualitative des pratiques conversationnelles en ligne des marques}

Nous avons mené une observation non participante de données publiées en ligne par les marques et par les consommateurs, inspirée de l'ethnographie virtuelle multi-sites (Bouillé et al., 2016 ; Hine, 2007). Pour mener une étude basée sur des données collectées en ligne, il est nécessaire de s'assurer qu'un volume suffisant de données soit disponible (Kozinets, 2010). Dans ce but, nous avons sélectionné des marques «connues» dans des secteurs où les internautes sont particulièrement actifs. En effet, les consommateurs qui interagissent avec les marques étant minoritaires (Campbell et al., 2014), nous avions plus de chances de pouvoir identifier des conversations de marque en choisissant des marques connues, puisqu'elles 
entrainent a priori un plus grand nombre de consommateurs que des marques peu connues. Pour identifier des marques «connues », nous avons croisé trois sources indiquant un certain niveau de notoriété : les marques les plus achetées ${ }^{1}$, les marques préférées ${ }^{2}$ et les marques ayant les plus fortes valeurs financières ${ }^{3}$.

De plus, pour améliorer encore les conditions de notre collecte, nous avons ciblé des secteurs d'activité où les internautes sont particulièrement actifs. Les secteurs des produits de grande consommation et de la mode comportant des consommateurs fortement engagés (Dessart et al., 2015), nous y avons choisi deux catégories de produits : les boissons non alcoolisées et les produits d'hygiène/beauté. Une troisième catégorie, l'automobile, a été choisie en raison de l'implication particulièrement forte des consommateurs au sein de communautés de marque en ligne (Hutter et al., 2013). Enfin, nous avons choisi deux marques dans chacune de ces trois catégories, dans le but d'observer une certaine diversité de situations, soit respectivement Carte Noire et Joker, Guerlain et Le Petit Marseillais, Audi et Peugeot.

Cette volonté de collecter un large éventail de situations d'interaction suit les principes d'une «observation naturaliste non systématique »(Goffman, 1973, p. 17). Cette méthode consiste à s'immerger dans un contexte donné - ici, celui des utilisateurs de médias sociaux qui conversent avec des marques, à collecter des données in situ sans intervenir, afin d'éviter de modifier le comportement des sujets, et à rechercher la diversité et l'intérêt des situations plutôt que leur exhaustivité. Nous avons ainsi réalisé des recherches sur les principaux médias sociaux (Facebook, Instagram, LinkedIn, Twitter, YouTube) et sur les sites des marques qui comportaient, pour certains, des sections de type blog (ex. : Peugeot, Carte Noire) et forum (ex. : Le Petit Marseillais). La collecte a été interrompue quand l'analyse du corpus a révélé une saturation théorique (Glaser et Strauss, 1967). L'unité d'analyse choisie, « une conversation », correspond au minimum à deux messages successifs dont l'un est rédigé pour le compte d'une marque. Parmi les 151 conversations collectées, 117 sont initiées par une marque et 34 sont initiées par un tiers. Ces conversations comprennent au total 5.214 commentaires $^{4}$, soit une

\footnotetext{
${ }^{1}$ Etude Brand Footprint Kantar Worldpanel, 2015. Sources : LSA. http://www.lsa-conso.fr/les-50-marques-les-plusachetees-en-france-sont,210031 et Brandfootprint : http://www.brandfootprint-ranking.com/\#/explore-the-data

${ }^{2}$ Etude Toluna pour Le Grand Livre des marques, 2015. Sources : Challenge http://www.challenges.fr/entreprise/20150130.CHA2665/quelles-sont-les-marques-preferees-des-francais.html et LSA http://www.lsa-conso.fr/quelles-sont-les-marques-preferees-des-francais, 199012

${ }^{3}$ Etude Brand Finance, 2015. Source : LSA http://www.lsa-conso.fr/les-marques-les-plus-puissantes-au-monde-eten-france-selon-brand-finance-infographie, 201790

4 Nombre estimé en fonction des fonctionnalités offertes par les différentes plates-formes. Le nombre de commentaires relevé a été soit celui indiqué sous la publication initiale (ex. : Facebook, LinkedIn, YouTube), soit celui relevé lors d'un comptage manuel (ex. : Instagram, Twitter). Notre estimation est basse en raison de
} 
moyenne de 34 commentaires par conversation $(\min =1 ; \max =931)$. Le contenu collecté représente 341 pages comprenant 137.292 mots.

Ces données ont fait l'objet d'une analyse sur deux niveaux. Le premier niveau a consisté à réaliser une analyse de contenu en codant, selon les cas : (1) la conversation dans son ensemble ; (2) un «tour de parole », c'est-à-dire l'intervention d'un participant depuis sa prise de parole jusqu'à ce qu'un autre la prenne à son tour (Sacks et al., 1974) $)^{5}$; (3) une «paire adjacente », c'est-à-dire deux tours de parole constituant un enchaînement (ex. : une question et sa réponse ; Schegloff et Sacks, 1973). Les unités sémantiques codées ont été identifiées lors de consultations répétées et approfondies des données. Elles ont été regroupées au sein de catégories et de sous-catégories, élaborées et organisées tout au long de l'analyse selon une démarche inductive (Bardin, 1977). Puis, des codes correspondant à des concepts issus de la théorie du face-work ont été utilisés, à savoir les FTA, atténuations et FFA, dans le but d'étudier le contexte de leur apparition.

Le deuxième niveau d'analyse a consisté à approfondir l'étude des conversations en suivant les principes de l'analyse conversationnelle (Schegloff et Sacks, 1973 ; Sacks, Schegloff et Jefferson, 1974 ; Kerbrat-Orecchioni, 2007). Développée dans le cadre de l'étude des interactions en face à face entre individus, l'analyse conversationnelle est un courant de recherche en linguistique qui s'intéresse aux plus petits détails de la conversation, pour mettre en évidence une structure de l'interaction (Goffman, 1973). Elle porte une attention particulière à la façon dont sont organisés les «tours de paroles» et leurs combinaisons en «paires adjacentes », concepts déjà mobilisés dans notre phase de codage.

Pour illustrer nos résultats, nous avons opté pour une transcription des conversations en ligne sur le modèle des transcriptions de conversations en face à face de l'analyse conversationnelle (ex. : Kerbrat-Orecchioni, 2007), plutôt que pour des copies d'écran. Ce format de présentation permet de respecter l'anonymat des internautes afin de se prémunir contre une utilisation non éthique de données publiques issues d'internet (Godes et Mayzlin, 2004 ; Kozinets, 2010). De plus, la numérotation des tours de parole permet de s'y référer aisément lors de la présentation des résultats.

l'affichage des commentaires sur les pages Facebook qui indiquait alors uniquement le nombre de commentaires mais pas les « réponses aux commentaires ».

${ }^{5}$ L'équivalent d'un tour de parole, sur les médias sociaux, peut correspondre à une publication ou un commentaire. 


\section{Entretiens semi-directifs auprès de consommateurs}

En complément de l'étude de données en ligne, nous avons mené des entretiens en face à face auprès de douze consommateurs, pour bénéficier d'une compréhension plus approfondie des perceptions des consommateurs vis-à-vis de la conversation de marque et dans un but de triangulation. De plus, notre étude s'intéressant aux internautes qui participent à des conversations de marque mais aussi à ceux, majoritaires, qui ne font que les observer, l'analyse du comportement en ligne des participants n'était pas suffisante pour comprendre les perceptions des spectateurs.

Nous avons fait appel à un échantillon de convenance en utilisant l'âge et le sexe comme critères de sélection. En effet, les caractéristiques démographiques peuvent induire des réactions particulières aux opérations marketing sur les médias sociaux (Campbell et al., 2014). Deux femmes et deux hommes ont ainsi été choisis au sein de chacune de ces trois tranches d'âge : 18 à 34 ans, 35 à 59 ans, 60 ans et plus. Une attention particulière a également été portée à la diversité des catégories socioprofessionnelles (Tableau 3).

\section{[Insérer Tableau 3]}

Les entretiens, semi-directifs, se sont appuyés sur un guide d'entretien organisé autour de trois thèmes: (1) la présence des marques sur les médias sociaux, (2) l'observation des échanges entre marques et internautes et (3) la participation éventuelle à de tels échanges. En fin d'entretien, des stimuli ont été présentés afin de faire réagir les répondants à différents types de conversation de marque, sous la forme de copies d'écran. Nous avons sélectionné quatre stimuli en fonction de la richesse des contenus et de la diversité des situations d'interaction. Chacun de ces stimuli représente une des catégories de notre typologie des conversations de marque (voir Tableau 1), respectivement : (1) une publication de la Caisse d'Epargne sur Facebook suivie de commentaires d'internautes ; (2) une discussion sur le site internet de co-création MyStarbucksIdea.com; (3) un tweet de Gaumont Pathé interpellant un internaute ; (4) un échange entre Free Mobile et un consommateur ayant besoin d'une assistance technique, sur Twitter. Notre collecte de données en ligne ayant porté sur des marques de produits, nous avons ici privilégié des marques de service, afin de renforcer la diversité des cas étudiés.

Les entretiens ont duré de 58 minutes à 1 heure et 34 minutes $(M=1 \mathrm{~h} 12 \mathrm{~min})$. Ils ont été enregistrés puis transcrits intégralement. Le corpus final représente 266 pages et 93.655 mots, soit une moyenne de 22 pages et 7.805 mots par entretien. Nous avons analysé les entretiens en utilisant un codage inductif. Les codes ont été déterminés au fur et à mesure de l'analyse, en 
fonction du sens émergeant des verbatims et sans être définis a priori en fonction de la littérature (Arnould et Wallendorf, 1994). L'analyse des entretiens a fait l'objet de triangulation entre les chercheurs. Ces derniers ont comparé et discuté leurs analyses, afin de parvenir à une compréhension commune (Goulding, 1999).

\section{Résultats}

Nos résultats montrent en quoi les marques s'approprient les règles du face-work dans leurs conversations en ligne et comment leurs FTA, atténuations et FFA génèrent des perceptions et réactions ambivalentes chez les consommateurs.

\section{Production et atténuation de FTA dans la conversation de marque : de la réticente à l'engagement}

Production et atténuation de FTA par les marques. Sur les médias sociaux, les marques cherchent l'engagement et la participation des consommateurs, notamment pour optimiser la qualité de la relation marque-consommateur (Hudson et al., 2015). Paradoxalement, cela se traduit fréquemment par la production d'actes de langage qui, selon la théorie du face-work, risquent de porter atteinte à la face de leur interlocuteur et donc de compromette la poursuite d'une interaction harmonieuse. Sur la base de la typologie des FTA de Brown et Levinson (1987), l'analyse des conversations de marque collectées montre deux formes de FTA associées à sept pratiques linguistiques (Tableau 4).

\section{[Insérer Tableau 4]}

Les marques utilisent fréquemment les call-to-actions, ces formules suggérant une action de la part des internautes (ex. : «Découvrez... »), prenant la forme de conseils ou s'apparentant même parfois à des ordres (ex. : «LIKEZ, COMMENTEZ ou PARTAGEZ»). Dans un autre registre, peut-être plus subtil, les marques «s'imposent» aux consommateurs par le biais de cadeaux ou de promesses (ex. : «sans surprise, vous serez conquise »). Au-delà de la volonté de susciter de l'engagement envers le produit ou la marque, les FTA ont parfois un rôle plus fonctionnel, dans le cas d'avertissements ou de requêtes, qui peuvent notamment guider les internautes dans la résolution de problèmes concernant le service client.

Par ailleurs, les FTA sont souvent accompagnés de formules qui, selon la théorie du face-work, visent à atténuer leur portée potentiellement négative. Sur la base de la typologie 
des atténuations de Brown et Levinson (1987), l'analyse de nos données permet d'identifier trois formes d'atténuations associées à six pratiques linguistiques (Tableau 5).

\section{[Insérer Tableau 5]}

Pour atténuer leurs FTA, les marques peuvent chercher à proposer plusieurs options plutôt que d'imposer une solution unique. Elles peuvent aussi choisir de dépersonnaliser ou de généraliser leur propos, ou encore émettre une suggestion par le biais d'une formule interrogative, plutôt qu'impérative (ex.: «Arriverez-vous à trouver le nombre exact d'oranges? » plutôt que «Trouvez le nombre exact d'oranges! »). Cependant, nous avons relevé des perceptions contrastées vis-à-vis de l'utilisation des FTA et de leurs atténuations.

Perception des FTA et de leur atténuation : réticence et engagement. Par nature, les FTA utilisés par les marques risquent d'entacher le sentiment de liberté des internautes en empiétant sur leur territoire (Goffman, 1973). En particulier, les call-to-actions des marques, le plus souvent des ordres selon notre typologie des FTA (voir Tableau 4), semblent effectivement exacerber les critiques des internautes, comme le montre le témoignage de Guillaume :

"C'est souvent des appels.(...) "Cliquez sur ça pour gagner un téléphone Samsung gratuit, il y en a que 400, dépêchez-vous", des choses comme ça. C'est juste pour que la personne elle clique et qu'elle reçoive après des messages de Samsung ou autre marque. (...) Pour moi c'est du vice tout ça, je sais pas comment dire. C'est pas très sain, voilà. Souvent c'est ça. "Clique sur ci, clique sur ça", c'est de l'impératif, voilà. Ca me répulse. Les gens ils cliquent s'ils ont envie, tu vois. »(Guillaume).

Les critiques de Guillaume sont particulièrement fortes lorsqu'il s'agit de «publications sponsorisées ${ }^{6}$ sur Facebook provenant de marques qu'il ne connait pas particulièrement. Dans la même veine, une conversation à l'initiative d'Audi sur sa page Facebook montre aussi que certains consommateurs acceptent mal les injonctions des marques sur les médias sociaux :

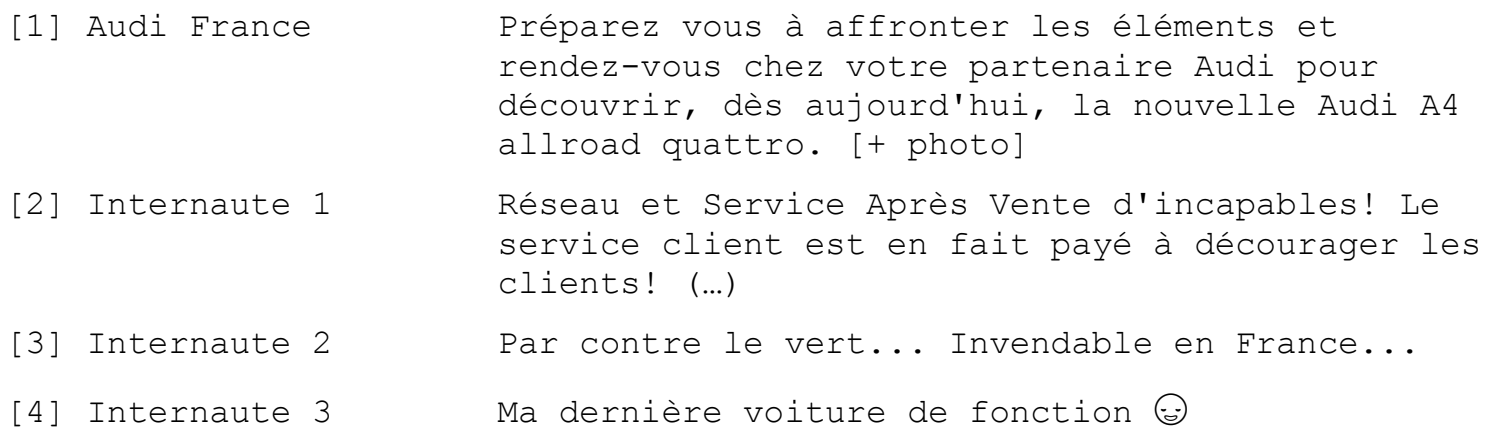

\footnotetext{
${ }^{6}$ Les publications sponsorisées sont des messages émis par les marques qui utilisent le même dispositif conversationnel que leurs publications habituelles sur les médias sociaux, mais peuvent toucher une audience plus large que les seuls abonnés de la marque, en fonction du budget alloué à leur diffusion.
} 
[5] Internaute 4

[6] Internaute 5

[7] Internaute 6
J'aime pas du tout la couleur...

Vert bouteille comme dans les années 80 yessss

J adore la couleur

Le message initial d'Audi, sur le mode impératif, contient un avertissement («préparezvous à... ») et un ordre («rendez-vous chez... »). Ses potentielles appréciations négatives ne sont ni atténuées, ni contrebalancées par un FFA. En l'occurrence, les internautes adressent des reproches à la marque (tour 2) et désapprouvent ouvertement le modèle présenté (tours 3 et 5). Ces réactions s'apparentent à un mécanisme de défense (Zemack-Rugar et al. 2017) face aux messages assertifs des marques. Cependant, les réactions des consommateurs sont rarement univoques. D'autres internautes réagissent ici de façon plus neutre (tour 4), ambigüe (6) ou favorable (7). Cette diversité des réactions laisse supposer que, face à un FTA, les internautes oscillent entre réticence et engagement selon leur familiarité avec la marque. Justine montre ainsi en quoi sa connaissance de la marque lui permet d'accepter ce mode conversationnel incitatif sans que cela heurte son désir de liberté :

«J'avoue que je ne suis pas une grande consommatrice.... Ah si tiens, j'ai rajouté, sur Facebook, oui, des créateurs de vêtements, parce que j'aimais bien. (...) Tu as l'impression que c'est plus toi qui décide. Et ça je préfère ouais. (...) Un petit truc une fois par semaine comme ça qui rappelle, qui dit: " ah oui, tiens c'est vrai, ils existent, tiens il y a un sac à main, tiens je vais aller voir la nouvelle collection de sacs à mains. » ça va juste me faire penser et puis après c'est à moi à cliquer et aller voir. Et puis j'oublie la première fois, et puis j'oublie la deuxième fois, et puis comme il y en a une par semaine... Une par semaine, à un moment donné, je vais finir par aller voir quand j'ai besoin $d u$ sac ou du...(...) Il ne faut pas que ce soit intrusif, sinon ça peut être contreproductif... On peut être tranquille sur Facebook avec nos amis, en paix quoi ». (Justine).

Ce témoignage montre qu'un FTA n'est pas forcément perçu comme intrusif quand l'internaute a choisi de suivre la marque au préalable et ne se considère donc pas dans une situation où elle risque de perdre la face. C'est aussi le cas de Marina, qui décrit l'intérêt qu'elle porte aux suggestions publiées par des musées qu'elle suit sur Facebook :

"Je suis abonnée à beaucoup de musées, par exemple à la Maison européenne de la photo, aussi à un musée à New York spécialisé dans la photo. Parce que j'aime bien savoir... Ou sinon à Tate Gallery, des choses comme ça (...). Comme ça je suis informée de ce qu'il se passe dans chaque galerie d'art par exemple (...) C'est pas envahissant, pas du tout. Et c'est très bien. C'est des contenus culturels par rapport aux artistes qui sont exposés dans les institutions, c'est tout. (...) Les musées nous convoquent pas, hein... C'est juste une suggestion. Pour s'amuser, pour s'instruire, c'est tout. (...) [Ça peut m'arriver de commenter], oui, si le sujet m'intéresse. Si je connais bien le sujet et que je vais pas dire de bêtises. Si je trouve que c'est important ou pertinent, oui. » (Marina). 
La répondante perçoit ici spontanément les messages incitatifs des marques comme des suggestions et les considère acceptables voire bienvenus. Le risque de perte de face causé par des FTA, fussent-ils incitatifs, semble ici limité dans la mesure où Marina est dans une relation de confiance voire de reconnaissance envers ces marques qui lui conseillent des sorties culturelles. Bien que les FTA, dans certaines situations, puissent être émis sans dommage, la théorie du face-work indique que, lorsqu'un risque de perte de face est présent, il est souhaitable de l'atténuer grâce à l'utilisation de formules adaptées. Dans les deux exemples suivants, les marques prennent certaines précautions pour atténuer leurs call-to-actions. Ainsi, dans un premier exemple, la marque Audi encourage les internautes à visionner une vidéo, sur YouTube :

Audi France

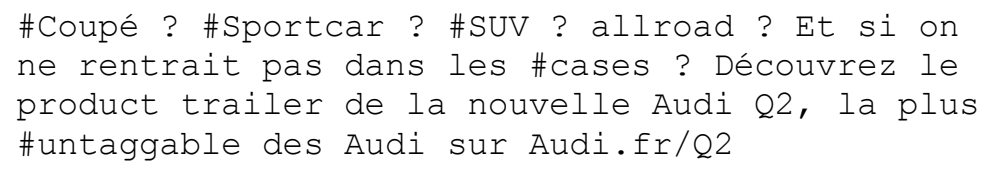

Dans un deuxième exemple, la marque Carte Noire incite à découvrir sa nouvelle gamme de capsules, sur Facebook :

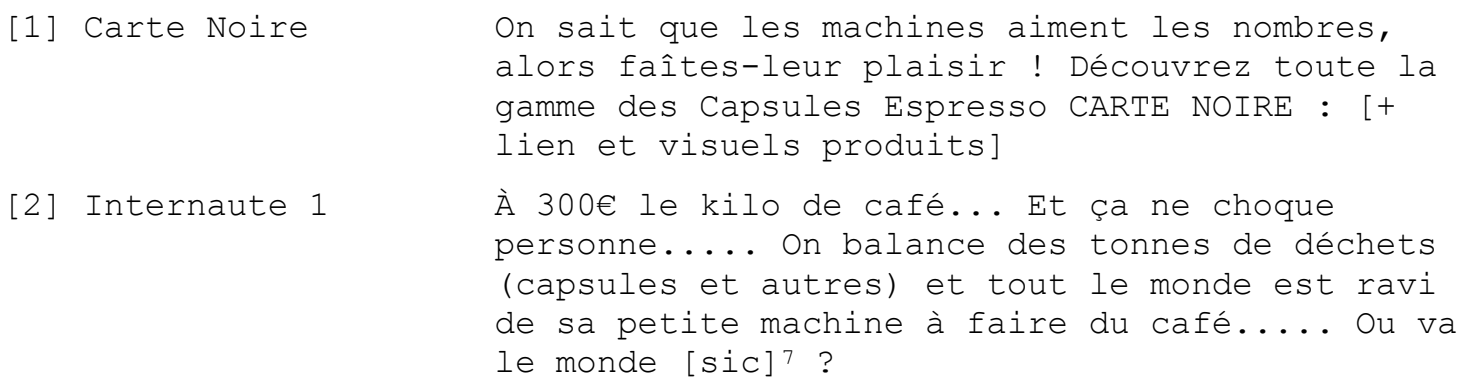

Les marques Audi et Carte Noire utilisent ici le mode impératif propre aux « ordres » de notre typologie (tableau 4), mais intègrent simultanément plusieurs formules qui viennent atténuer les effets potentiellement négatifs de ces incitations. Dans le premier exemple, Audi fait preuve de prudence en utilisant la forme interrogative «Et si ?». De plus, le pronom « on » marque une dépersonnalisation qui protège les interlocuteurs en leur permettant d'être inclus dans le sujet. Ces deux postures, prudence et protection, sont caractéristiques des atténuations de FTA (voir Tableau 5). Dans le deuxième exemple, Carte Noire utilise le même type d'atténuation («on sait que »). Face à ce FTA atténué, la critique de l'internaute (tour 2) peut constituer une réaction à la perception d'une menace (Chen, 2015 ; Peña et Brody, 2014). Mais l'internaute semble aussi chercher à atténuer son propre FTA en utilisant des formules de

\footnotetext{
${ }^{7}$ Tous les verbatims sont reproduits tels quels.
} 
dépersonnalisation («Et ça ne choque personne») et de généralisation («tout le monde»). Ainsi, le respect des règles du face-work permet à l'échange de se dérouler malgré tout, en suivant ici le principe de liberté de la communication interpersonnelle. Ce phénomène est notamment observé lorsque les interlocuteurs jugent utile de préserver leur relation (Mauney et Jeon, 2014).

\section{Production de FFA dans la conversation de marque : de la gratitude au scepticisme}

Production de FFA par les marques. Dans leurs conversations avec les consommateurs, les marques utilisent toute la gamme disponible des FFA. Sur la base de la typologie des FFA de Brown et Levinson (1987), l'analyse des conversations de marque montre trois formes de FFA associées à huit pratiques linguistiques (Tableau 6).

\section{[Insérer Tableau 6]}

Les marques peuvent chercher à coopérer avec les consommateurs, pour témoigner d'une forme de proximité, ou tenter d'augmenter la satisfaction des internautes en se montrant compréhensives et en faisant preuve de sympathie (ex. : «Heureux que Joko vous plaise et vous fasse rire $(-) »)$. Enfin, leurs actes valorisants sont fréquents, sous des formes qui vont des signes d'attention à des traits d'humour, en passant par des formules d'approbation et des compliments (ex. : «C'est parce que nous avons des supers ambassadeurs !! »).

Perception des FFA: gratitude et scepticisme. Nos résultats révèlent des perceptions ambivalentes des consommateurs face à la production de FFA, censée répondre au besoin de reconnaissance de l'interlocuteur. L'analyse de nos données montre que les marques mettent en œuvre des stratégies de valorisation des internautes qui génèrent souvent un sentiment de gratitude. Par exemple, Sylvie apprécie le fait que la marque Starbucks manifeste un intérêt sincère aux contributions des internautes sur son site de co-création :

"J'aime bien. Je trouve ça très sympa. Tu peux voir les idées qui sont en action. J'imagine que ça veut dire en train de se construire (...) C'est bien, c'est sympa. Parce qu'il y a des gens qui ont réagi et la marque a réagi. C'est ça qui me plait en fait (...) Là le fait que le mec, dans sa réponse, soit assez modéré, il dit « on y a pensé, mais c'est un peu complexe, on ne sait pas trop, mais on va en reparler à nos équipes » et puis les commentaires des gens, là je marche à fond. J'ai l'impression de savoir ce qui se passe. (...). C'est plus clair (...), je n'ai pas l'impression qu'on se fout de ma gueule (rires). (...) J'ai l'impression d'être impliquée (...). J'ai l'impression d'être dans le processus. » (Sylvie). 
Ce témoignage illustre l'utilisation de FFA de type «inclusion » (voir Tableau 6) et rejoint l'idée que les techniques visant à valoriser les consommateurs, notamment en les incluant au sein d'une communauté, suscitent des attitudes positives chez ceux qui ont proposé des idées à la marque (Fombelle et al., 2016). De plus, Sylvie témoigne de sa satisfaction en tant qu'observatrice des interactions, du fait des réponses à la fois «modérées » et inclusives du représentant de la marque. A l'instar du Petit Marseillais sur sa page Facebook, comme dans l'exemple présenté ci-dessous, les marques répondent parfois individuellement aux internautes en les valorisant par des FFA contenant des « approbations » et des « compliments » :

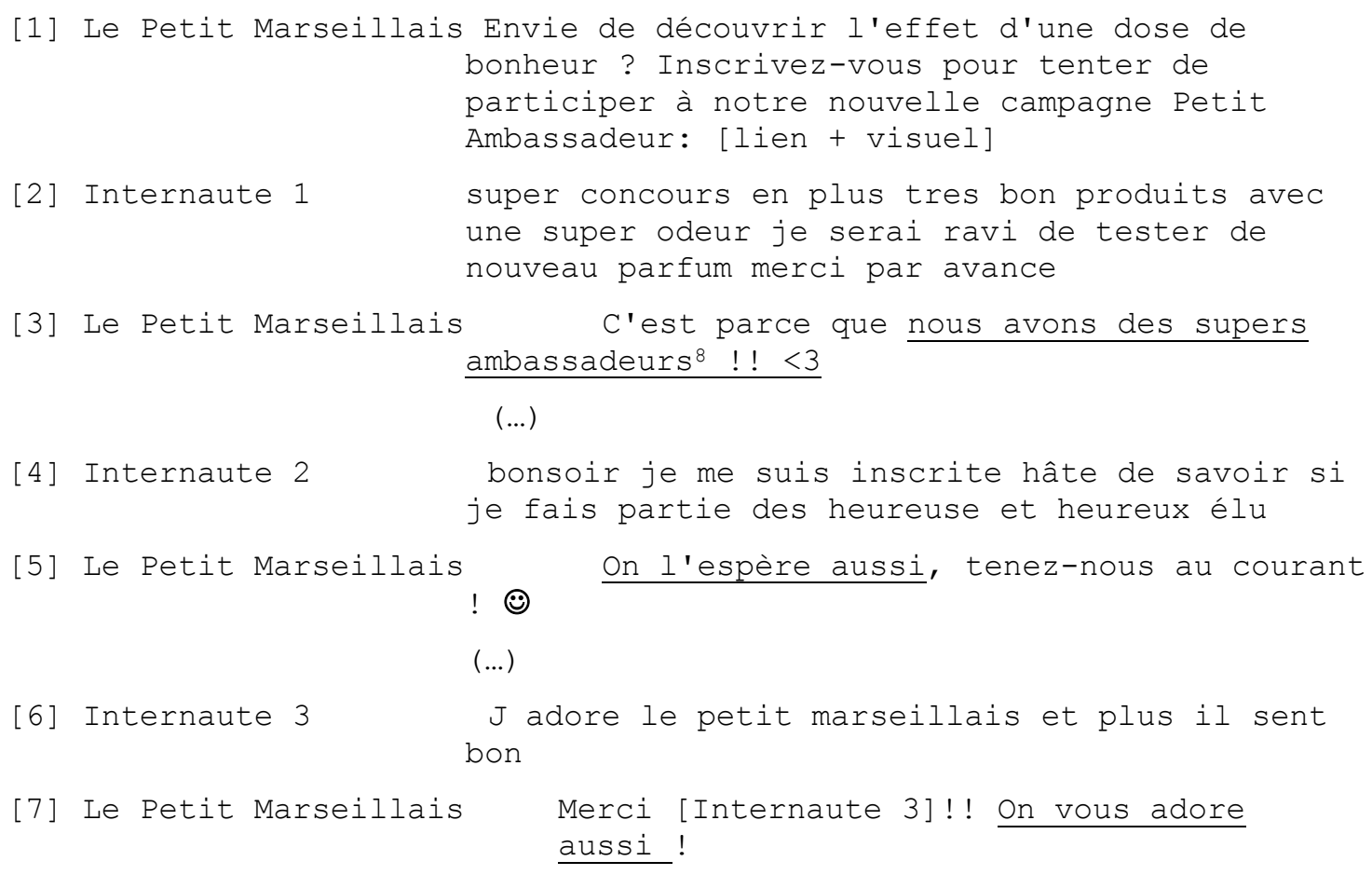

Cette conversation débute par une formule interrogative permettant à la marque d'atténuer le FTA produit immédiatement après («Inscrivez-vous »). Puis, la marque utilise des formules flattant les internautes (tours 3 et 7) et leur montre son approbation et son soutien (tour 5). Ces actes de langage ont pour fonction «d'augmenter» la face des internautes (Kerbrat-Orecchioni, 2010). Ils contribuent à renforcer le climat favorable de la conversation et à pérenniser l'ordre de l'interaction. Toutefois, les FFA ne génèrent pas toujours des appréciations positives. En particulier, la déférence - une forme de politesse très appuyée observée dans plusieurs interventions de marques - semble sujette à caution. En effet, des réponses longues et détaillées de la part des marques contrastent parfois avec la brièveté, voire la dureté, des messages

\footnotetext{
${ }^{8}$ Les passages sont soulignés par les auteurs.
} 
d'internautes. Ces échanges peuvent susciter un doute quant à la sincérité de la marque ou provoquer une forme d'exaspération, comme l'explique ici Justine :

«Les gens ils voient qu'il y a des cons qui postent des commentaires stupides, et la façon dont répond la marque, ça peut être tout à son honneur aussi (...). Ce n'est pas la peine d'être mielleux ou de passer des heures avec un con. Une réponse très polie, mais un petit peu qui remet les pendules à l'heure aussi. Les autres sont contents de voir ça aussi, que tu as quelqu'un d'un peu intelligent derrière, que c'est pas que du mielleux : "Ah bon, on est vraiment désolé ". Ça peut être une réponse ferme, si l'entreprise a raison, parce que des fois elle a raison, juste une petite réponse posée. (...) Je pense que ça ne sert à rien d'essayer de répondre pendant des heures aux boulets. »(Justine).

Justine apprécie les conversations directes et sincères, sans faux-semblants. Ce témoignage rejoint l'idée que les réponses trop valorisantes peuvent s'apparenter à de la manipulation (Wang et Chaudhry, 2018). Au prisme du face-work, ces messages peuvent être considérés comme de «l'hyperpolitesse », définie par la présence de marqueurs excessifs par rapport aux normes en vigueur dans un contexte donné (Kerbrat-Orecchioni, 2010). Ceci rejoint l'idée que le non-respect des normes conversationnelles est potentiellement préjudiciable pour les marques, même s'il s'agit de FFA. De plus, notre analyse montre que l'hyperpolitesse des marques peut survenir aussi bien en réponse à des messages négatifs qu'à des messages positifs. Par exemple, Lionel commente une interaction entre le compte officiel des cinémas Gaumont Pathé et un internaute en montrant une forme d'agacement vis-à-vis de la marque :

"Qu'une marque vienne s'immiscer dans mon univers pour essayer de créer un lien soidisant sympathique "qu'est-ce que tu as pensé du film? ", là ça va trop loin. (...) Là, je veux qu'on me laisse tranquille. Ce que j'ai pensé du film, je le garde pour moi, je le partage éventuellement avec mes amis, si j'ai envie de le faire, mais je n'ai pas envie qu'une enseigne, qu'une marque me demande ce que j'ai pensé du film. (...) C'est mon espace, c'est mon univers. C'est aussi une forme de pudeur. Je n'ai pas envie d'exposer aux yeux de tout le monde ce que je pense de tout et de... Pour moi, ce ne serait pas très... D'ailleurs, me poser la question c'est m'inciter à répondre alors que je n'ai absolument pas envie de répondre. Je trouve ça gênant. » (Lionel).

Lionel juge l'intervention de la marque déplacée et intrusive, bien que les manifestations d'attention et de sympathie soient censées être des FFA (voir Tableau 6). Ce témoignage montre que la nature commerciale de la marque vient entraver les règles de la conversation. Les interventions de la marque, même constituées de FFA, peuvent être mal interprétées à la fois par le destinataire et les spectateurs car elles sont généralement considérées comme ayant un objectif commercial. Ce constat complète les recherches précédentes qui ont montré que certains consommateurs peuvent juger de façon négative les marques qui font état d'une politesse particulièrement appuyée dans leurs publicités (Sundar et Cao, 2020). 
En résumé, nos résultats révèlent des réactions ambivalentes des internautes au cours de leurs interactions avec les marques, ce qui fait écho aux travaux de Goffman (1967) pour qui un compliment ou une récompense peuvent plonger le bénéficiaire dans la confusion ou dans un sentiment de mal-être, tandis qu'un individu peut rester positif et apprécier une réponse négative si elle est perçue comme adaptée au contexte.

\section{Discussion et conclusion}

Sur la base d'une analyse de 151 conversations en ligne impliquant six marques dans trois secteurs d'activité et d'une série d'entretiens semi-directifs avec 12 consommateurs, cette recherche contribue à enrichir la littérature relative aux interactions entre marques et consommateurs en étendant la théorie du face-work au contexte où l'un des interlocuteurs est une marque. De plus, nos résultats nous amènent à formuler des recommandations dans les domaines de la gestion de la marque et du community management.

\section{Contribution à la littérature sur les interactions en ligne entre marques et consommateurs}

Notre recherche identifie la diversité des FTA, des atténuations et des FFA utilisés par les marques, qui rejoint la typologie de Brown et Levinson (1987) et met en évidence l'ambivalence que peuvent générer ces différentes pratiques linguistiques. Nous montrons tout d'abord que l'utilisation des FTA par les marques peut avoir des conséquences négatives sur la poursuite de l'interaction. De plus, conformément aux règles de la conversation interpersonnelle, les marques ont la possibilité d'atténuer ces FTA ou de produire des FFA, pour répondre aux besoins de liberté et de reconnaissance des individus et voir l'échange se poursuivre. En complément, nos résultats révèlent que les FTA émis par des marques ne génèrent pas forcément de perceptions négatives, notamment si l'internaute entretient des relations particulières avec une marque et apprécie d'être sollicité par une marque qu'il estime.

En ce qui concerne les FFA utilisés par les marques, ils génèrent plutôt un sentiment de gratitude, mais ils peuvent également induire un sentiment de suspicion aux yeux des internautes qui peuvent, dans un contexte commercial, y percevoir un manque de sincérité de la part de la marque. Par ailleurs, alors que les recherches en marketing n'avaient jusque-là identifié la présence de FFA que dans le cas de la réduction des effets négatifs des FTA (Fombelle et al., 2016), notre étude montre que les FFA sont également produits en réponse à des commentaires positifs des internautes, c'est-à-dire dans un contexte déjà favorable à la bonne tenue de l'interaction. 
En mettant l'accent sur les normes conversationnelles et en révélant les conséquences de leur respect ou de leur transgression par les marques, nous répondons à l'appel de Smith et Rose (2020), qui constataient que le rôle des normes d'usages dans les relations en ligne n'avait pas été suffisamment étudié et constituait une voie de recherche importante. Notre recherche complète la littérature en examinant le modèle conversationnel des échanges de propos en ligne à la lumière de la théorie du face-work et en prenant en compte à la fois les participants et les spectateurs. En nous intéressant à la fois aux FTA, à leur atténuation et aux FFA, nous répondons aussi à l'appel de Villarroel Ordenes et al. (2019) pour qui les chercheurs devraient mener des analyses détaillées des messages des marques qui comportent simultanément plusieurs intentions.

\section{Contribution à la théorie du face-work}

Notre analyse montre que les interactions entre marques et internautes intègrent des stratégies et des mécanismes du face-work similaires à ceux observés dans les interactions entre individus. Cependant, nos résultats suggèrent également que la relation à la marque ainsi que la nature symbolique des marques, associée au contexte commercial des échanges, peuvent venir questionner les principes du face-work.

Comme exposé précédemment, notre recherche montre que les FTA des marques n'entravent pas nécessairement la poursuite de l'interaction. Ce phénomène peut être expliqué par la nature de la relation avec la marque. En effet, les internautes pourraient se plier aux injonctions de la marque ou du moins ne pas s'en offusquer en considérant que le risque de « perdre la face » avec une marque qu'ils connaissent est moindre que le risque de perdre la face avec une marque inconnue. Ces résultats concordent avec le fait que les internautes tolèrent un langage informel de la part des marques qu'ils connaissent, mais pas de la part d'une marque inconnue (Gretry et al., 2017). Ils révèlent aussi que les conditions d'application des règles du face-work prévalent également dans le cas de la conversation de marque : les stratégies de facework peuvent être plus relâchées quand il préexiste une certaine familiarité entre les interlocuteurs, le risque de perte de face étant réduit en présence de personnes proches (Brown et Levinson, 1987).

Cependant, la nature symbolique d'une marque en tant qu'interlocuteur peut aussi expliquer les appréciations positives des FTA dans certaines circonstances. Les internautes feraient alors abstraction de la présence d'une « vraie » personne (ex. : un community manager) aux commandes des comptes de la marque en vertu du phénomène de «suspension d'incrédulité », qui voit les consommateurs participer à la création de sens en acceptant la part 
fictionnelle du récit de marque (Benmoussa et Maynadier, 2013). En se mettant en situation de dialogue avec «la marque », en tant qu'objet symbolique et non personne humaine, les internautes pourraient moins ressentir le risque de perte de face inhérent à une relation interpersonnelle et par conséquent mieux accepter la transgression de règles du face-work de la part des marques, notamment lorsqu'elles produisent des FTA. Cette situation rappelle celle où les internautes, notamment les plus jeunes, préfèrent interagir avec un agent virtuel plutôt qu'avec un humain, afin de conserver un sentiment de contrôle (Köhler et al., 2011).

En ce qui concerne les FFA, nos résultats montrent qu'ils peuvent parfois générer des perceptions négatives. Certes, les internautes impliqués envers une marque peuvent se sentir flattés et surpris du fait que leur objet de dévotion s'adresse à eux (Schamari et Schaefers, 2015). Cependant, notre recherche montre que les actes de valorisation produits par les marques peuvent être perçus comme peu sincères, du fait qu'ils se déroulent dans le cadre d'une communication dont les consommateurs sont conscients des objectifs sous-jacents. Ainsi, audelà du phénomène d'hyperpolitesse (Kerbrat-Orecchioni, 2010), déjà connu dans le cas des interactions entre individus, les réactions négatives d'internautes face aux FFA des marques peuvent être expliquées par le contexte commercial des échanges, qui peut laisser planer un doute sur les intentions de la marque (Wang et Chaudhry, 2018).

En conclusion, cette recherche contribue à la littérature sur le face-work en montrant que, d'une part, les règles du face-work s'appliquent globalement aux pratiques conversationnelles des marques sur les médias sociaux mais que, d'autre part, la relation à la marque, la nature symbolique de la marque et le contexte commercial sous-jacent peuvent expliquer des réactions et perceptions positives vis-à-vis des FTA, et négatives vis-à-vis des FFA.

\section{Implications managériales}

Cette recherche permet aux praticiens de mieux comprendre la perception de trois principales pratiques linguistiques (FTA, atténuation de FTA et FFA) dans la conversation de marque, offrant ainsi de nouvelles perspectives à la construction des relations marques-consommateurs et à la pratique du community management basées sur une utilisation éclairée des FTA, atténuations et FFA. Nous proposons ainsi un outil d'aide à la décision sous forme de recommandations pour chacune des formes conversationnelles présentées dans la première partie de cet article (Tableau 7).

[Insérer Tableau 7] 
(1) Dans les conversations des marques hôtes-proactives, les marques ont la main et peuvent adapter leur style d'interaction selon leur identité. En revanche, la production de FTA doit être parcimonieuse. Si les FTA semblent pouvoir fonctionner dans le cas de messages destinés aux internautes déjà abonnés à la page d'une marque, nos résultats montrent qu'ils devraient être évités dans les publications sponsorisées, qui touchent une plus large audience. Dans ces circonstances, les community managers doivent privilégier des formules peu intrusives (ex. : une suggestion plutôt qu'un ordre), en accompagnant les FTA d'atténuations (ex. : formules interrogatives plutôt qu'impératives) et/ou de FFA.

(2) Le dispositif conversationnel d'une marque hôte-réactive semble propice à répondre au besoin de reconnaissance des consommateurs. En effet, les espaces communautaires sont par nature inclusifs et coopératifs, comme par exemple les espaces de co-création où les consommateurs peuvent proposer des idées de nouveaux produits et ont la possibilité de s'exprimer et de voir leur contribution reconnue. Pour répondre davantage au besoin de reconnaissance des consommateurs, les marques peuvent utiliser des FFA, comme des signes d'approbation et des témoignages de sympathie.

(3) Dans le cas d'une conversation d'une marque invitée-proactive, la marque doit prendre certaines précautions car elle intervient de façon non sollicitée au sein d'espaces qu'elle ne maîtrise pas. Le style d'interaction devra donc être généralement factuel et d'une tonalité neutre, évitant à la fois les FTA trop intrusifs et les FFA trop appuyés. Les interventions au sein de ces espaces doivent veiller à minimiser la perception par les consommateurs d'une intrusion sur leur territoire. Il conviendrait donc ici de privilégier les atténuations, par exemple en offrant des options et en posant des questions.

(4) Enfin, dans les conversations où la marque est invitée-réactive, il est souhaitable de s'adapter à la situation et à la problématique de l'internaute. En particulier, dans le cadre d'une assistance client ou de la réponse à des questions, mieux vaut privilégier un style directif et efficace, qui par conséquent peut comporter des FTA non atténués comme des conseils et des requêtes, voire des ordres, si cela peut aboutir à la résolution rapide du problème soulevé par un client.

L'utilisation des stratégies de face-work dans les interactions marques-consommateurs en ligne aurait pu paraitre assez naturelle puisque, derrière les publications de la marque se trouve généralement un être humain, par exemple un community manager, conditionné par son expérience personnelle des règles de la communication interindividuelle. Pourtant, force est de constater que toutes ces interactions ne se déroulent pas toujours de façon satisfaisante pour les marques. Les règles du face-work pourraient finalement ne pas être si bien connues ni 
maîtrisées par les praticiens dans un contexte commercial. Nos résultats invitent donc les praticiens à mieux prendre en compte les principes de la conversation interpersonnelle. Des formations de sensibilisation à ces règles pourraient être proposées à tous les salariés en contact avec les consommateurs sur internet.

\section{Limites et voies de recherches}

Le caractère exploratoire de cette étude ouvre de nouvelles perspectives de recherche. En effet, après avoir identifié la diversité des pratiques linguistiques des marques et analysé l'ambivalence des perceptions et réactions qu'elles génèrent, il serait important d'identifier les variables individuelles et contextuelles qui pourraient jouer un rôle sur l'usage de ces procédés. Il serait par exemple utile de mieux comprendre comment la perception des FTA et des FFA peut être altérée par: (1) le type d'internautes concernés (participants ou spectateurs), (2) le sujet de la conversation (plus ou moins lié à des aspects commerciaux), et (3) le type de relation entretenue avec la marque. En particulier, sur ce dernier point, des recherches futures pourraient tenter de mieux comprendre les conditions dans lesquelles le risque de perte de face est plus ou moins important (ex. : familiarité ou attachement à la marque) pour ainsi compléter les critères proposés par Brown et Levinson (1987), à savoir la relation de pouvoir ainsi que les facteurs sociaux et culturels des interlocuteurs. Notre recherche se situant dans un contexte français, d'autres études pourraient être menées pour prendre en compte les différences culturelles, ce qui compléterait ainsi les travaux de Hudson et al. (2015) qui montrent comment les traits culturels viennent modifier l'influence des interactions et la qualité de la relation marque-consommateur.

Nos résultats ayant montré des réactions contrastées des internautes vis-à-vis de la façon dont les marques se conforment plus ou moins aux normes conversationnelles, de futures recherches pourraient plus spécifiquement s'intéresser à la façon dont l'application de ces règles impacte la perception de la marque. Suite aux recherches qui montrent que les interactions entre clients et personnel en contact sont perçues différemment selon la cohérence entre la personnalité des salariés et celle de la marque (Sirianni et al., 2013), il pourrait être intéressant de comprendre en quoi le respect plus ou moins strict des règles du face-work par les marques permet d'exprimer et/ou d'enrichir leur personnalité. Plus précisément, des recherches pourraient tenter de comprendre comment les FTA, les atténuations ou les FFA viennent modifier ou façonner l'image de compétence et de cordialité, deux facettes importantes de la personnalité des marques (Fournier et Alvarez, 2012). Dans cette perspective, il serait également utile d'approfondir l'analyse de chacune des stratégies du face-work (FTA, 
atténuation de FTA et FFA) en introduisant les différentes nuances identifiées dans la littérature et présentées dans cette recherche dans les tableaux 4, 5 et 6 .

Par ailleurs, bien que notre recherche s'appuie sur des médias sociaux les plus utilisés, il serait important de développer des recherches sur les conversations de marque sur des platesformes ayant plus récemment émergé, comme par exemple TikTok. De plus, les usages des internautes comme ceux des marques se développent aussi dans le cadre de conversations privées, sur des plates-formes comme Snapchat, Messenger ou WhatsApp. Il parait donc important de mieux comprendre comment les règles conversationnelles s'appliquent dans un contexte où le regard de l'autre ne fait plus partie de l'équation. Dans le contexte des interactions privées avec une marque, ces règles se trouvent-elles renforcées, ou au contraire relâchées, par rapport aux interactions en public?

De plus, dans la mesure où le marketing fait face au développement incessant de nouveaux outils interactifs, il serait intéressant de poursuivre cette analyse de la conversation de marque à la lumière de la théorie du face-work dans le contexte des objets connectés et des dispositifs de réalité augmentée ou virtuelle. Nos résultats pourraient notamment être vérifiés dans le cadre des conversations automatisées grâce à l'intelligence artificielle, comme par exemple dans le cas des chatbots, ces agents conversationnels installés au sein des messageries instantanées sous forme d'interfaces textuelles ou accompagnant les enceintes connectées sous forme d'interfaces vocales. Ces tendances comptent en effet parmi celles qui vont fortement impacter les pratiques marketing, en particulier sur les médias sociaux (Appel et al., 2020). Ainsi, dans la continuité de cette étude des pratiques linguistiques dans la conversation de marque, il serait désormais important, pour les chercheurs comme pour les praticiens, d'intégrer de façon plus globale l'analyse de la conversation écrite ou orale entre marques et consommateurs.

\section{Remerciements}

Nous remercions tout d'abord le rédacteur en chef, le rédacteur en chef associé et les trois lecteurs anonymes pour leurs commentaires positifs et constructifs, qui nous ont permis d'améliorer notre manuscrit tout au long du processus d'évaluation. Cette recherche a été réalisée dans le cadre du laboratoire COACTIS (Universités Lumière Lyon 2 et Jean Monnet Saint-Etienne) et de la Chaire Marques \& Valeurs de l'IAE Paris-Sorbonne. Nous en remercions les membres respectifs pour la richesse de leurs échanges. Nous remercions aussi la société Entrecom, membre de la Chaire Marques \& Valeurs, pour son soutien à cette recherche. 


\section{Références}

Appel G, Grewal L, Hadi R, et al. (2020) The future of social media in marketing. Journal of the Academy of Marketing Science 48(1): 79-95. DOI: 10.1007/s11747-019-00695-1.

Arnould EJ et Wallendorf M (1994) Market-oriented ethnography: interpretation building and marketing strategy formulation. Journal of Marketing Research 31(4): 484-504. DOI: $10.1177 / 002224379403100404$.

Bardin L (1977) L'analyse de contenu. Paris: Presses universitaires de France.

Bedijs K, Held G et Maaß C (2014) Introduction: face work and social media. In: Bedijs K, Held G, et Maaß C (eds) Face work and social media. Münster: LIT, pp. 9-28.

Benmoussa F-Z et Maynadier B (2013) Brand storytelling: entre doute et croyance. Une étude des récits de la marque Moleskine. Décisions Marketing: 119-128. DOI: 10.7193/dm.070.119.128.

Berthelot-Guiet K (2011) Extension du domaine de la conversation: discours de marque et publicitarité. Communication \& langages 3(169): 77-86. DOI: 10.4074/S0336150011003073.

Bouillé J, Basso F et Robert-Demontrond P (2016) La rhétorique incarnée de l'activisme consumériste au regard de la théorie de la métaphore conceptuelle : étude exploratoire et perspectives de recherche. Recherche et Applications en Marketing 31(2): 86-112. DOI: $10.1177 / 0767370115622951$.

Breitsohl J, Kunz WH et Dowell D (2015) Does the host match the content? A taxonomical update on online consumption communities. Journal of Marketing Management 31(9): 1040-1064. DOI: 10.1080/0267257X.2015.1036102.

Brown P et Levinson S (1987) Politeness: some universals in language use. Studies in interactional sociolinguistics 4. Cambridge: Cambridge University Press.

Campbell C, Ferraro C et Sands S (2014) Segmenting consumer reactions to social network marketing. European Journal of Marketing 48(3): 432-452. DOI: 10.1108/EJM-03-20120165.

Chen GM (2015) Losing face on social media: threats to positive face lead to an indirect effect on retaliatory aggression through negative affect. Communication Research 42(6): 819838. DOI: $10.1177 / 0093650213510937$.

Chen K-J, Lin J-S, Choi JH, et al. (2015) Would you be my friend? An examination of global marketers' brand personification strategies in social media. Journal of Interactive Advertising 15(2): 97-110. DOI: 10.1080/15252019.2015.1079508.

Cruz RE, Leonhardt JM et Pezzuti T (2017) Second person pronouns enhance consumer 
involvement and brand attitude. Journal of Interactive Marketing 39: 104-116. DOI: 10.1016/j.intmar.2017.05.001.

De Montety C et Patrin-Leclère V (2011) La conversion à la conversation: le succès d'un succédané. Communication \& langages 3(169): 23-37. DOI: 10.4074/S0336150011003036.

Dessart L, Veloutsou C et Morgan-Thomas A (2015) Consumer engagement in online brand communities: a social media perspective. Journal of Product \& Brand Management 24(1): 28-42. DOI: 10.1108/JPBM-01-2015-0790.

Drouet M (2011) De «la communication » à « la conversation » : vers un nouveau paradigme en publicité? Communication \& langages 2011(169): 39. DOI: 10.4074/S0336150011003048.

Fombelle PW, Bone SA et Lemon KN (2016) Responding to the 98\%: face-enhancing strategies for dealing with rejected customer ideas. Journal of the Academy of Marketing Science 44(6): 685-706. DOI: 10.1007/s11747-015-0469-y.

Fournier S et Alvarez C (2012) Brands as relationship partners: Warmth, competence, and inbetween. Journal of Consumer Psychology 22(2), 177-185. DOI: 10.1016/J.JCPS.2011.10.003.

Fournier S et Avery J (2011) The uninvited brand. Business Horizons 54(3): 193-207. DOI: 10.1016/j.bushor.2011.01.001.

Glaser BG et Strauss AL (1967) The discovery of grounded theory: strategies for qualitative Rresearch. Aldine. DOI: 10.2307/2575405.

Godes D et Mayzlin D (2004) Using online conversations to study word-of-mouth communication. Marketing Science 23(4): 545-560. DOI: 10.1287/mksc.1040.0071.

Goffman E (1955) On face-work: an analysis of ritual elements in social interaction. Psychiatry: Journal for the Study of Interpersonal Processes 18(3): 213-231. DOI: $10.1162 / 15241730360580159$.

Goffman E (1967 [1974]) Les Rites d'interaction. Paris : Editions de Minuit.

Goffman E (1973) La Mise En Scène de La Vie Quotidienne. 2: Les Relations En Public. Paris: Editions de Minuit.

Goulding C (1999) Consumer research, interpretive paradigms and methodological ambiguities. European Journal of Marketing 33(9/10): 859-873. DOI: 10.1108/03090569910285805.

Gretry A, Horváth C, Belei N, et al. (2017) 'Don’t pretend to be my friend!' When an informal brand communication style backfires on social media. Journal of Business Research 74: 77-89. DOI: 10.1016/j.jbusres.2017.01.012. 
Griffiths M et Mclean R (2015) Unleashing corporate communications via social media: A UK study of brand management and conversations with customers. Journal of Customer Behaviour 14(2): 147-162. DOI: 10.1362/147539215X14373846805789.

Hamilton M, Kaltcheva VD et Rohm AJ (2016) Hashtags and handshakes: consumer motives and platform use in brand-consumer interactions. Journal of Consumer Marketing 33(2): 135-144. DOI: 10.1108/JCM-04-2015-1398.

Hine C (2007) Multi-sited ethnography as a middle range methodology for contemporary STS. Science, Technology, \& Human Values 32(6). 652-671. DOI: 10.1177/0162243907303598.

Homburg C, Ehm L et Artz M (2015) Measuring and managing consumer sentiment in an online community environment. Journal of Marketing Research 52(5): 629-641. DOI: 10.1509/jmr.11.0448.

Hudson S, Huang L, Roth MS, et al. (2015) The influence of social media interactions on consumer-brand relationships: a three-country study of brand perceptions and marketing behaviors. International Journal of Research in Marketing 1(33): 27-41. DOI: 10.1016/j.ijresmar.2015.06.004.

Hutter K, Hautz J, Dennhardt S, et al. (2013) The impact of user interactions in social media on brand awareness and purchase intention: the case of MINI on Facebook. Journal of Product \& Brand Management 22(5/6): 342-351. DOI: 10.1108/JPBM-05-2013-0299.

Kerbrat-Orecchioni C (1992) Les interactions verbales. Tome II. Paris: A. Colin.

Kerbrat-Orecchioni C (1998) La notion d'interaction en linguistique : origine, apports, bilan. Langue française 117: 51-67. DOI: 10.3406/lfr.1998.6241.

Kerbrat-Orecchioni C (2005) Le discours en interaction. Paris: Armand Colin,.

Kerbrat-Orecchioni C (2007) L'analyse du discours en interaction: quelques principes méthodologiques. Limbaje si comunicare IX: 13-32.

Kerbrat-Orecchioni C (2010) L'impolitesse en interaction: aperçus théoriques et étude de cas. Lexis Special (2): 35-60.

Kerssen-Griep J, Trees AR et Hess JA (2008) Attentive facework during instructional feedback: key to perceiving mentorship and an optimal learning environment. Communication Education 57(3): 312-332. DOI: 10.1080/03634520802027347.

Köhler CF, Rohm AJ, de Ruyter K et al. (2011) Return on interactivity: the impact of online agents on newcomer adjustment. Journal of Marketing 75(2): 93-108. DOI: 10.1509/jmkg.75.2.93.

Kozinets RV. (2010) Netnography: doing ethnographic research online. Londres: 
Sage Publications.

Kumar A, Bezawada R, Rishika R, et al. (2016) From social to sale: the effects of firmgenerated content in social media on customer behavior. Journal of Marketing 80(1): 7-25. DOI: 10.1509/jm.14.0249.

Labrecque LI (2014) Fostering consumer-brand relationships in social media environments: the role of parasocial interaction. Journal of Interactive Marketing 28(2): 134-148. DOI: 10.1016/j.intmar.2013.12.003.

Li X (Shirley), Chan KW et Kim S (2018) Service with emoticons: how customers interpret employee use of emoticons in online service encounters. Journal of Consumer Research 45(March): 1-50. DOI: 10.1093/jcr/ucy016.

Liu Y et Shrum LJ (2002) What is interactivity and is it always such a good thing? Implications of definition, person, and situation for the influence of interactivity on advertising effectiveness. Journal of Advertising 31(4): 53-64. DOI: 10.1080/00913367.2002.10673685.

Mauney S et Jeon L (2014) "I love you but I disagree": politeness and politics in computermediated discourse. Rice Working Papers in Linguistics, 5. http://hdl.handle.net/1911/78540.

Michel G (2017) Au coeur de la marque - 3ème éd.: les clés du management des marques. Paris: Dunod.

Peña $\mathrm{J}$ et Brody N (2014) Intentions to hide and unfriend Facebook connections based on perceptions of sender attractiveness and status updates. Computers in Human Behavior 31(1): 143-150. DOI: 10.1016/j.chb.2013.10.004.

Sacks H, Schegloff EA et Jefferson G (1974) A simplest systematics for the organization of turn taking for conversation. Language 50(4): 696-735. DOI: 10.2307/412243.

Schamari J et Schaefers T (2015) Leaving the home turf: how brands can use webcare on consumer-generated platforms to increase positive consumer engagement. Journal of Interactive Marketing 30: 20-33. DOI: 10.1016/j.intmar.2014.12.001.

Schegloff EA et Sacks H (1973) Opening up closings. Semiotica 8(4): 289-327.

Simon F et Tossan V (2018) Does brand-consumer social sharing matter? A relational framework of customer engagement to brand-hosted social media. Journal of Business Research 85: 175-184. DOI: 10.1016/j.jbusres.2017.12.050.

Sirianni NJ, Bitner, MJ, Brown, SW et Mandel, N (2013) Branded service encounters: Strategically aligning employee behavior with the brand positioning. Journal of Marketing 77(6), 108-123. DOI:_10.1509/jm.11.0485. 
Smith AN, Fischer E et Yongjian C (2012) How does brand-related user-generated content differ across YouTube, Facebook, and Twitter? Journal of Interactive Marketing 26(2): 102-113. DOI: 10.1016/j.intmar.2012.01.002.

Smith LW et Rose RL (2020) Service with a smiley face: emojional contagion in digitally mediated relationships. International Journal of Research in Marketing 37(2): 301-319. DOI: 10.1016/j.ijresmar.2019.09.004.

Sundar A et Cao ES (2020) Punishing politeness: the role of language in promoting brand trust. Journal of Business Ethics 164: 39-60. DOI: 10.1007/s10551-018-4060-6.

Van Dolen WM, Dabholkar PA et De Ruyter K (2007) La satisfaction envers les discussions en ligne de clients: l'influence des attributs technologiques perçus, des caractéristiques du groupe de discussion et du style de communication du conseiller. Recherche et Applications en Marketing 22(3): 339-358. DOI: 10.1177/076737010702200306.

Van Noort G et Willemsen LM (2012) Online damage control: the effects of proactive versus reactive webcare interventions in consumer-generated and brand-generated platforms. Journal of Interactive Marketing 26(3): 131-140. DOI: 10.1016/j.intmar.2011.07.001.

Villarroel Ordenes F, Grewal D, Ludwig S, et al. (2019) Cutting through content clutter: how speech and image acts drive consumer sharing of social media brand messages. Journal of Consumer Research 45(5): 988-1012. DOI: 10.1093/jcr/ucy032.

Wang Y et Chaudhry A (2018) When et how managers' responses to online reviews affect subsequent reviews. Journal of Marketing Research 55(2). American Marketing Association: 163-177. DOI: 10.1509/jmr.15.0511.

Zemack-Rugar Y, Moore SG et Fitzsimons GJ (2017) Just do it! Why committed consumers react negatively to assertive ads. Journal of Consumer Psychology 27(3): 287-301. DOI: 10.1016/j.jcps.2017.01.002. 
Annexe 1 - Principaux travaux sur la conversation de marque

\begin{tabular}{|c|c|c|c|c|c|c|}
\hline Article & Initiative & Lieu & Plateforme & Population & Focus & Résultats \\
\hline $\begin{array}{l}\text { Villarroel } \\
\text { Ordenes et al. } \\
\text { (2019) }\end{array}$ & $\begin{array}{l}\text { Marque } \\
\text { proactive }\end{array}$ & $\begin{array}{l}\text { Marque } \\
\text { hôte }\end{array}$ & $\begin{array}{l}\text { Facebook } \\
\text { Twitter }\end{array}$ & Participants & $\begin{array}{l}\text { Partage des publications; } \\
\text { nature de l'acte de langage }\end{array}$ & $\begin{array}{l}\text { Les messages des marques émotionnels ou } \\
\text { informatifs sont plus partagés par les } \\
\text { consommateurs que les messages directifs. }\end{array}$ \\
\hline $\begin{array}{l}\text { Simon et } \\
\text { Tossan (2018) }\end{array}$ & $\begin{array}{l}\text { Marque } \\
\text { proactive }\end{array}$ & $\begin{array}{l}\text { Marque } \\
\text { hôte }\end{array}$ & Facebook & $\begin{array}{l}\text { Audience } \\
\text { multiple } \\
\text { (non } \\
\text { spécifiée) }\end{array}$ & $\begin{array}{l}\text { Engagement des } \\
\text { consommateurs ; valeur du } \\
\text { partage }\end{array}$ & $\begin{array}{l}\text { Les consommateurs qui trouvent une forme de } \\
\text { gratification dans l'interaction avec la marque } \\
\text { sont plus engagés envers sa page. }\end{array}$ \\
\hline $\begin{array}{l}\text { Li et al. } \\
(2018)\end{array}$ & $\begin{array}{l}\text { Marque } \\
\text { proactive }\end{array}$ & $\begin{array}{l}\text { Marque } \\
\text { hôte }\end{array}$ & Facebook & Spectateurs & $\begin{array}{l}\text { Satisfaction des } \\
\text { consommateurs ; utilisation } \\
\text { d'émoticônes }\end{array}$ & $\begin{array}{l}\text { Les consommateurs sont plus satisfaits quand les } \\
\text { marques utilisent des émoticônes, si les normes } \\
\text { relationnelles "communautaires" sont valorisées, }\end{array}$ \\
\hline $\begin{array}{l}\text { Cruz et al. } \\
\text { (2017) }\end{array}$ & $\begin{array}{l}\text { Marque } \\
\text { proactive }\end{array}$ & $\begin{array}{l}\text { Marque } \\
\text { hôte }\end{array}$ & $\begin{array}{l}\text { Facebook } \\
\text { Blogs }\end{array}$ & $\begin{array}{l}\text { Spectateurs } \\
\text { Audience } \\
\text { multiple } \\
\text { (non } \\
\text { spécifiée) }\end{array}$ & $\begin{array}{l}\text { Implication et attitude des } \\
\text { consommateurs ; utilisation } \\
\text { de pronoms à la seconde } \\
\text { personne }\end{array}$ & $\begin{array}{l}\text { L'utilisation de pronoms à la deuxième personne } \\
\text { dans les messages des marques a un impact } \\
\text { positif sur l'implication des consommateurs et } \\
\text { leur attitude envers la marque. }\end{array}$ \\
\hline $\begin{array}{l}\text { Gretry et al. } \\
(2017)\end{array}$ & $\begin{array}{l}\text { Marque } \\
\text { proactive }\end{array}$ & $\begin{array}{l}\text { Marque } \\
\text { hôte }\end{array}$ & Facebook & Spectateurs & $\begin{array}{l}\text { Confiance envers la marque } \\
\text { style de communication de la } \\
\text { marque }\end{array}$ & $\begin{array}{l}\text { Un langage informel augmente la confiance } \\
\text { envers la marque lorsque les consommateurs } \\
\text { connaissent la marque. }\end{array}$ \\
\hline $\begin{array}{l}\text { Kumar et al. } \\
\text { (2016) }\end{array}$ & $\begin{array}{l}\text { Marque } \\
\text { proactive }\end{array}$ & $\begin{array}{l}\text { Marque } \\
\text { hôte }\end{array}$ & $\begin{array}{l}\text { Non } \\
\text { dévoilé } \\
\text { (type } \\
\text { Facebook) }\end{array}$ & $\begin{array}{l}\text { Audience } \\
\text { multiple } \\
\text { (non } \\
\text { spécifiée) }\end{array}$ & $\begin{array}{l}\text { Ventes, intensité de la } \\
\text { relation, rentabilité ; volume } \\
\text { et réceptivité des contenus }\end{array}$ & $\begin{array}{l}\text { Plus la marque interagit avec les consommateurs, } \\
\text { plus l'impact est positif sur les ventes, l'intensité } \\
\text { de la relation e la rentabilité du client. }\end{array}$ \\
\hline $\begin{array}{l}\text { Wang et } \\
\text { Chaudhry } \\
(2018)\end{array}$ & $\begin{array}{l}\text { Marque } \\
\text { réactive }\end{array}$ & $\begin{array}{l}\text { Marque } \\
\text { invitée }\end{array}$ & Tripadvisor & Spectateurs & $\begin{array}{l}\text { Valence des commentaires; } \\
\text { stratégie de réponse aux avis }\end{array}$ & $\begin{array}{l}\text { La réponse des managers aux avis négatifs à un } \\
\text { effet positif sur la valence des avis suivants, } \\
\text { d'autant plus si la réponse est personnalisée. }\end{array}$ \\
\hline $\begin{array}{l}\text { Fombelle et al. } \\
(2016)\end{array}$ & $\begin{array}{l}\text { Marque } \\
\text { réactive }\end{array}$ & $\begin{array}{l}\text { Marque } \\
\text { hôte }\end{array}$ & Forums & Participants & $\begin{array}{l}\text { Partage d'idées ; amélioration } \\
\text { de la face }\end{array}$ & $\begin{array}{l}\text { Les entreprises peuvent réduire l'effet négatif du } \\
\text { rejet des idées des consommateurs en créant une } \\
\text { identité de groupe ou en s'excusant en public. }\end{array}$ \\
\hline
\end{tabular}




\begin{tabular}{|c|c|c|c|c|c|c|}
\hline $\begin{array}{l}\text { Homburg et } \\
\text { al. (2015) }\end{array}$ & $\begin{array}{l}\text { Marque } \\
\text { réactive }\end{array}$ & $\begin{array}{l}\text { Marque } \\
\text { hôte }\end{array}$ & Forums & Participants & $\begin{array}{l}\text { Sentiment envers la marque ; } \\
\text { niveau d'engagement de } \\
\text { l'entreprise }\end{array}$ & $\begin{array}{l}\text { Plus les entreprises participent aux conversations, } \\
\text { plus l'attitude envers elles est positive, jusqu'à un } \\
\text { certain point. }\end{array}$ \\
\hline $\begin{array}{l}\text { Schamari et } \\
\text { Schaefers } \\
(2015)\end{array}$ & $\begin{array}{l}\text { Marque } \\
\text { réactive }\end{array}$ & $\begin{array}{l}\text { Marque } \\
\text { hôte et } \\
\text { invitée }\end{array}$ & $\begin{array}{l}\text { Facebook } \\
\text { Forums }\end{array}$ & Spectateurs & $\begin{array}{l}\text { Engagement des } \\
\text { consommateurs ; stratégie de } \\
\text { réponse aux commentaires }\end{array}$ & $\begin{array}{l}\text { La réponse des marques aux commentaires } \\
\text { positifs sur des forums gérés par des internautes a } \\
\text { un impact positif sur leur intention d'engagement. }\end{array}$ \\
\hline $\begin{array}{l}\text { Breitsohl et al. } \\
\text { (2015) }\end{array}$ & $\begin{array}{l}\text { Marque } \\
\text { proactive } \\
\text { et réactive }\end{array}$ & $\begin{array}{l}\text { Marque } \\
\text { hôte et } \\
\text { invitée }\end{array}$ & Forums & Participants & $\begin{array}{l}\text { Fréquence de publication des } \\
\text { consommateurs ; bénéfices } \\
\text { perçus }\end{array}$ & $\begin{array}{l}\text { Le bénéfice perçu des échanges a un effet plus } \\
\text { positif sur la fréquence de publication au sein des } \\
\text { communautés gérées par les marques (vs. par les } \\
\text { consommateurs). }\end{array}$ \\
\hline $\begin{array}{l}\text { Van Noort et } \\
\text { Willemsen, } \\
(2012)\end{array}$ & $\begin{array}{l}\text { Marque } \\
\text { réactive }\end{array}$ & $\begin{array}{l}\text { Marque } \\
\text { hôte et } \\
\text { invitée }\end{array}$ & $\mathrm{B} \log \mathrm{s}$ & Spectateurs & $\begin{array}{l}\text { Evaluation de la marque } \\
\text { stratégie de réponse aux } \\
\text { commentaires }\end{array}$ & $\begin{array}{l}\text { Les réponses "réactives" des marques aux } \\
\text { commentaires négatifs entrainent une évaluation } \\
\text { plus positive de la marque que lorsqu'il n'y a pas } \\
\text { de réponse. }\end{array}$ \\
\hline $\begin{array}{l}\text { Smith et al., } \\
\text { (2012) }\end{array}$ & $\begin{array}{l}\text { Non } \\
\text { précisé }\end{array}$ & $\begin{array}{l}\text { Non } \\
\text { précisé }\end{array}$ & $\begin{array}{l}\text { Facebook } \\
\text { Twitter } \\
\text { YouTube }\end{array}$ & Participants & $\begin{array}{l}\text { Volume du UGC ; type de } \\
\text { média social ; stratégie de } \\
\text { présence de la marque }\end{array}$ & $\begin{array}{l}\text { Le volume de contenu généré par les utilisateurs } \\
\text { est plus important quand les marques offrent des } \\
\text { opportunités et des raisons d'être contactées. }\end{array}$ \\
\hline $\begin{array}{l}\text { Cette } \\
\text { recherche }\end{array}$ & $\begin{array}{l}\text { Marque } \\
\text { proactive } \\
\text { et réactive }\end{array}$ & $\begin{array}{l}\text { Marque } \\
\text { hôte et } \\
\text { invitée }\end{array}$ & $\begin{array}{l}\text { Facebook } \\
\text { Twitter } \\
\text { Instagram } \\
\text { LinkedIn } \\
\text { YouTube } \\
\text { Blogs } \\
\text { Forums }\end{array}$ & $\begin{array}{l}\text { Spectateurs } \\
\text { et } \\
\text { participants }\end{array}$ & $\begin{array}{l}\text { Respect des normes } \\
\text { conversationnelles par les } \\
\text { marques ; comportement et } \\
\text { perceptions des internautes }\end{array}$ & $\begin{array}{l}\text { Les principes du face-work (FTA, atténuations, } \\
\text { FFA) transparaissent dans la conversation de } \\
\text { marque. La relation à la marque ainsi que la } \\
\text { nature symbolique et commerciale de la marque } \\
\text { expliquent des réactions positives aux FTA et des } \\
\text { réactions négatives aux FFA. }\end{array}$ \\
\hline
\end{tabular}


Figure 1 - Les différentes stratégies de face-work selon le risque de perte de face (adapté de Brown et Levinson, 1987, et Kerbrat-Orecchioni, 1992)

Si le risque de perte de Si le risque de perte face est fort de face est faible

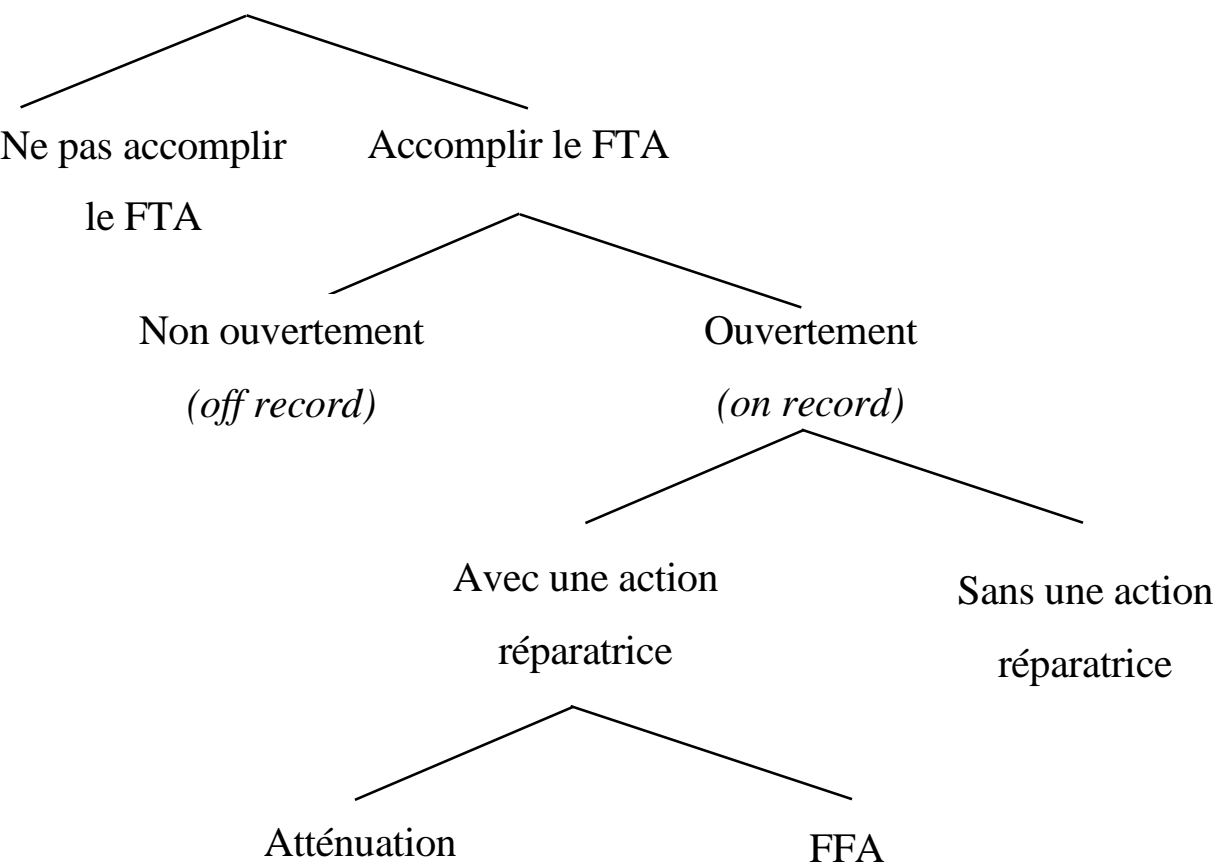


Tableau 1 - Les différentes formes de conversation de marque

\begin{tabular}{|c|c|c|}
\hline \multirow{2}{*}{$\begin{array}{l}\text { Initiative de la } \\
\text { conversation }\end{array}$} & \multicolumn{2}{|l|}{ Lieu de la conversation } \\
\hline & $\begin{array}{l}\text { Marque hôte qui converse sur des } \\
\text { espaces en ligne qu'elle maîtrise }\end{array}$ & $\begin{array}{l}\text { Marque invitée qui converse sur des } \\
\text { espaces en ligne qui ne lui } \\
\text { appartiennent pas }\end{array}$ \\
\hline $\begin{array}{l}\text { Marque proactive qui } \\
\text { initie la conversation }\end{array}$ & $\begin{array}{l}\text { 1. Conversation d'une marque hôte- } \\
\text { proactive } \\
\text { Publication d'une marque sur ses } \\
\text { pages sur les médias sociaux et } \\
\text { commentaires des internautes. } \\
\text { (ex. : Gretry et al. 2017; Kumar et al. } \\
\text { 2016) }\end{array}$ & $\begin{array}{l}\text { 3. Conversation d'une marque } \\
\text { invitée-proactive } \\
\text { Publication d'une marque au sein des } \\
\text { communautés en ligne ou vers des } \\
\text { comptes des consommateurs qui ne } \\
\text { s'adressent pas à elle. } \\
\text { (ex. : Breitsohl et al., 2015; Wang et } \\
\text { Chaudhry, 2018) }\end{array}$ \\
\hline $\begin{array}{l}\text { Marque réactive qui } \\
\text { intervient dans une } \\
\text { conversation initiée par } \\
\text { un consommateur }\end{array}$ & $\begin{array}{l}\text { 2. Conversation d'une marque hôte- } \\
\text { réactive } \\
\text { Réponse d'une marque à la publication } \\
\text { d'un consommateur au sein d'une page } \\
\text { communautaire gérée par la marque. } \\
\text { (ex. : Fombelle et al., 2016; Homburg } \\
\text { et al., 2015) }\end{array}$ & $\begin{array}{l}\text { 4. Conversation d'une marque } \\
\text { invitée-réactive } \\
\text { Réponse d'une marque aux messages } \\
\text { des internautes adressés à la marque } \\
\text { depuis leurs propres comptes. } \\
\text { (ex. : Schamari et Schaefers, 2015; } \\
\text { Van Noort et Willemsen, 2012) }\end{array}$ \\
\hline
\end{tabular}


Tableau 2 - Situations conversationnelles étudiées

\begin{tabular}{|c|c|c|c|c|}
\hline Méthode & Focus & Forme conversationnelle & Secteur & Marque \\
\hline Observation en ligne & $\begin{array}{l}\text { Stratégies de } \\
\text { face-work des marques ; } \\
\text { Comportement des } \\
\text { internautes participants }\end{array}$ & $\begin{array}{l}\text { Hôte-proactive; } \\
\text { Hôte-réactive ; } \\
\text { Invitée-réactive }\end{array}$ & $\begin{array}{l}\text { Alimentation; Automobile; } \\
\text { Hygiène-Beauté }\end{array}$ & $\begin{array}{l}\text { Carte Noire ; Joker ; } \\
\text { Audi ; Peugeot ; Le Petit } \\
\text { Marseillais ; Guerlain }\end{array}$ \\
\hline Entretiens & $\begin{array}{l}\text { Perceptions des internautes } \\
\text { (participants et spectateurs) }\end{array}$ & $\begin{array}{l}\text { Hôte-proactive ; } \\
\text { Hôte-réactive ; } \\
\text { Invitée-proactive } \\
\text { Invitée-réactive }\end{array}$ & $\begin{array}{l}\text { Banque ; Cinéma ; } \\
\text { Télécommunication ; } \\
\text { Restauration rapide ; Divers }\end{array}$ & $\begin{array}{l}\text { Caisse d'Epargne ; Gaumont- } \\
\text { Pathé ; Free Mobile ; } \\
\text { Starbucks ; citations } \\
\text { spontanées } \\
\text { (ex. : Apple, Décathlon, BNP) }\end{array}$ \\
\hline
\end{tabular}


Tableau 3 - Profil des répondants

\begin{tabular}{lllll}
\hline Prénom & Age & $\begin{array}{l}\text { Situation } \\
\text { familiale }\end{array}$ & $\begin{array}{l}\text { Niveau } \\
\text { d'études }\end{array}$ & Profession \\
\hline Alexis & 42 & Divorcé & $\mathrm{BEP}$ & Employé municipal \\
Antoine & 28 & En couple & $\mathrm{Bac}+5$ & Ingénieur \\
Carole & 61 & Célibataire & $\mathrm{Bac}+3$ & Bibliothécaire \\
Denis & 68 & Marié & $\mathrm{Bac}+5$ & Cadre retraité \\
Guillaume & 39 & Divorcé & $\mathrm{CAP}$ & Artisan peintre \\
Judith & 69 & Mariée & $\mathrm{Bac}+2$ & Assistante retraitée \\
Justine & 30 & Célibataire & $\mathrm{Bac}+5$ & Officier de marine \\
Lionel & 32 & Célibataire & $\mathrm{Bac}+5$ & Commerçant \\
Marc & 77 & Marié & $\mathrm{Bac}+5$ & Cadre retraité \\
Marina & 38 & En couple & $\mathrm{Bac}+3$ & Guide touristique \\
Sabine & 29 & Célibataire & $\mathrm{Bac}+3$ & Employée RH \\
Sylvie & 51 & Mariée & $\mathrm{Bac}$ & $\begin{array}{l}\text { Employée } \\
\text { administrative }\end{array}$ \\
\hline
\end{tabular}

${ }^{9}$ Les prénoms ont été modifiés. 
Tableau 4 - Les FTA (Face Threatening Acts) utilisés par les marques

\begin{tabular}{|c|c|c|}
\hline $\begin{array}{l}\text { Stratégies de } \\
\text { FTA }{ }^{10}\end{array}$ & $\begin{array}{l}\text { Pratiques } \\
\text { linguistiques }\end{array}$ & Exemples \\
\hline \multirow[t]{5}{*}{ Incitation (a) } & Avertissement & $\begin{array}{l}\text { - Bienvenue chez les Petits Ambassadeurs. Attention toutefois à } \\
\text { l'orthographe par respect pour la marque et pour le sérieux du } \\
\text { site } \odot . \text { Bonne journée (Le Petit Marseillais, forum) }\end{array}$ \\
\hline & Conseil & $\begin{array}{l}\text { - Internaute : J'ai essayé et j'aurais aimé que le gel douche ait } \\
\text { un parfum un peu plus fort car on ne le sent pas. } \\
\text { - Le Petit Marseillais : Si vous pensez qu'il y a un problème de } \\
\text { qualité avec l'un de nos produits, n'hésitez pas à contacter notre } \\
\text { service consommateurs. (Le Petit Marseillais, Facebook) }\end{array}$ \\
\hline & Ordre & $\begin{array}{l}\text { - MAINTENANT C'EST A VOUS ! Tentez de gagner une séance } \\
\text { de ciné privée avec vos amis : LIKEZ, COMMENTEZ ou } \\
\text { PARTAGEZ votre baiser préféré ! (Carte Noire, Facebook) }\end{array}$ \\
\hline & Requête & $\begin{array}{l}\text { - Pouvez-vous nous transmettre en DM }{ }^{11} \text { vos coordonnées et } \\
\text { numéro de dossier svp? (Audi, Twitter) }\end{array}$ \\
\hline & Suggestion & $\begin{array}{l}\text { - A l'occasion de la journée mondiale du bonheur, Le Petit } \\
\text { Marseillais vous invite à découvrir la nouvelle gamme Les P'tits } \\
\text { Bonheurs! (Le Petit Marseillais, blog) }\end{array}$ \\
\hline \multirow[t]{2}{*}{ Imposition (b) } & Cadeau & $\begin{array}{l}\text { - Le Petit Marseillais a décidé de vous accompagner et de se } \\
\text { glisser dans vos valises pour vos week-ends et vacances au } \\
\text { soleil (Le Petit Marseillais, Facebook) }\end{array}$ \\
\hline & Promesse & $\begin{array}{l}\text { - Sans surprise, vous serez conquise. Disponible dans notre } \\
\text { boutique en ligne (Guerlain, Facebook) }\end{array}$ \\
\hline
\end{tabular}

(a) Les incitations des marques visent à faire réaliser une action aux internautes. Les incitations tendent à réduire la liberté des internautes, qui peuvent se sentir contraints de réaliser l'action évoquée.

(b) Les impositions consistent à faire des offres non sollicitées aux internautes. Ces offres empiètent sur le territoire des internautes, lesquels peuvent se sentir redevables envers la marque qui s'impose à eux.

\footnotetext{
${ }^{10}$ Les listes des stratégies de FTA, d'atténuations, de FFA ainsi que les pratiques linguistiques associées sont adaptées de Brown and Levinson (1987).

${ }^{11}$ Direct message (message privé)
} 
Tableau 5 - Les atténuations de FTA (Face Threatening Act mitigation) utilisées par les marques

\begin{tabular}{|c|c|c|}
\hline $\begin{array}{l}\text { Stratégies } \\
\text { d'atténuation } \\
\text { des FTA }\end{array}$ & $\begin{array}{l}\text { Pratiques } \\
\text { linguistiques }\end{array}$ & Exemples \\
\hline \multirow[t]{2}{*}{ Liberté (a) } & Excuses & $\begin{array}{l}\text { - Bonjour [Internaute], nous sommes désolés de ne revenir vers } \\
\text { vous que maintenant. Pourriez-vous nous en dire davantage? } \\
\text { Merci. (Audi, Twitter). }\end{array}$ \\
\hline & Offrir des options & $\begin{array}{l}\text { - Bonjour [Internaute] N'hésitez pas à nous recontacter ou à } \\
\text { vous rapprocher de votre partenaire. (Audi, LinkedIn) }\end{array}$ \\
\hline \multirow[t]{2}{*}{ Protection (b) } & Dépersonnalisation & $\begin{array}{l}\text { - Movember commence aujourd'hui. Voici } 3 \text { excellentes façons } \\
\text { de soutenir la cause (Carte Noire, Facebook). }\end{array}$ \\
\hline & Généralisation & $\begin{array}{l}\text { - Audi S5 Coupé : vous devinez en un coup d'œil son } \\
\text { tempérament sportif. (Audi, Instagram) }\end{array}$ \\
\hline \multirow[t]{2}{*}{ Prudence (c) } & Esquive & $\begin{array}{l}\text { - Internaute :Oh @AudiFrance t'as choisi ton camp } ?^{12} \\
\text { - Audi France : On a tranché : une voiture allemande et une } \\
\text { victoire française. (Audi, Twitter) }\end{array}$ \\
\hline & Question & $\begin{array}{l}\text { - Arriverez-vous à trouver le nombre exact d'oranges? Attention } \\
\text { les yeux, ça va vite! (Joker, Facebook) }\end{array}$ \\
\hline
\end{tabular}

(a) La stratégie de liberté consiste à redonner la main aux internautes, par exemple en reconnaissant une erreur et/ou offrant le choix entre différentes alternatives.

(b) La stratégie de protection permet aux internautes de décider s'ils sont destinataires ou sujets d'un message qui contiendrait un potentiel FTA, en s'appuyant sur une formulation ambiguë.

(c) La stratégie de prudence consiste à s'exprimer de façon détournée pour éviter toute situation embarrassante ou perte de face que pourrait entrainer une formulation plus directe.

${ }^{12}$ Référence à un match de football France-Allemagne, le jour même. 
Tableau 6 - Les FFA (Face Flattering Acts) utilisés par les marques

\begin{tabular}{|c|c|c|}
\hline $\begin{array}{l}\text { Stratégies de } \\
\text { FFA }\end{array}$ & $\begin{array}{l}\text { Pratiques } \\
\text { linguistiques }\end{array}$ & Exemples \\
\hline \multirow[t]{2}{*}{ Coopération (a) } & Inclusion & $\begin{array}{l}\text { - Essayez de vous décrire en un seul mot. Impossible vous } \\
\text { dites? L'Audi Q2 est comme vous (Audi, Twitter) }\end{array}$ \\
\hline & Optimisme & $\begin{array}{l}\text { - On croise les doigts [Internaute]! } \\
\text { (Le Petit Marseillais, Facebook) }\end{array}$ \\
\hline \multirow[t]{2}{*}{ Satisfaction (b) } & Compréhension & $\begin{array}{l}\text { - Nous comprenons votre interrogation. } \\
\text { (Carte Noire, Facebook) }\end{array}$ \\
\hline & Sympathie & $\begin{array}{l}\text { - Heureux que Joko vous plaise et vous fasse rire @) } \\
\text { (Joker, Facebook) }\end{array}$ \\
\hline \multirow[t]{4}{*}{ Valorisation (c) } & Approbation & $\begin{array}{l}\text { - Très bon choix [Internaute] } \odot) \\
\text { (Le Petit Marseillais, Facebook) }\end{array}$ \\
\hline & Attention & $\begin{array}{l}\text { - Bonjour, nous sommes soucieux de la qualité des prestations } \\
\text { délivrées dans le réseau Peugeot, et regrettons les } \\
\text { désagréments que vous avez vécus dans l'utilisation de votre } \\
\text { Peugeot. (Peugeot, Facebook) }\end{array}$ \\
\hline & Compliments & $\begin{array}{l}\text { - C'est parce que nous avons des supers ambassadeurs !! } \\
\text { (Le Petit Marseillais, Facebook) }\end{array}$ \\
\hline & Humour & $\begin{array}{l}\text { - On donne notre langue au Lion dites-nous tout! } \\
\text { (Peugeot, Twitter) }\end{array}$ \\
\hline
\end{tabular}

(a) La stratégie de coopération consiste à faire participer les internautes à la narration ou à l'action et/ou à instaurer un climat positif entre eux et la marque.

(b) La stratégie de satisfaction consiste à maximiser la satisfaction perçue des internautes au cours de la conversation, en faisant preuve de soutien et en se montrant cordial.

(c) La stratégie de valorisation consiste à mettre en valeur les internautes de façon plus ou moins directe, dans le but d'obtenir une attitude favorable de leur part. 
Tableau 7 - Recommandations de pratiques linguistiques dans les quatre formes conversationnelles

\begin{tabular}{|c|c|c|c|c|}
\hline & $\begin{array}{l}\text { (1) Marque hôte- } \\
\text { proactive }\end{array}$ & $\begin{array}{l}\text { (2) Marque hôte- } \\
\text { réactive }\end{array}$ & $\begin{array}{l}\text { (3) Marque } \\
\text { invitée-proactive }\end{array}$ & $\begin{array}{l}\text { (4) Marque } \\
\text { invitée-réactive }\end{array}$ \\
\hline $\begin{array}{l}\text { Stratégies de } \\
\text { face-work à } \\
\text { privilégier }\end{array}$ & $\begin{array}{l}\text { FFA } \\
\text { Atténuations } \\
\text { Eviter les FTA } \\
\text { non atténués }\end{array}$ & $\begin{array}{l}\text { FFA (ex. : attention } \\
\text { portée aux } \\
\text { internautes) }\end{array}$ & $\begin{array}{l}\text { Atténuations } \\
\text { (ex. : offrir des } \\
\text { options) }\end{array}$ & $\begin{array}{l}\text { FTA non } \\
\text { atténués } \\
\text { (ex. : conseils, } \\
\text { requêtes) }\end{array}$ \\
\hline
\end{tabular}

